\title{
Fire Protection Analysis of The Orfalea College of Business Building (\#3)
}

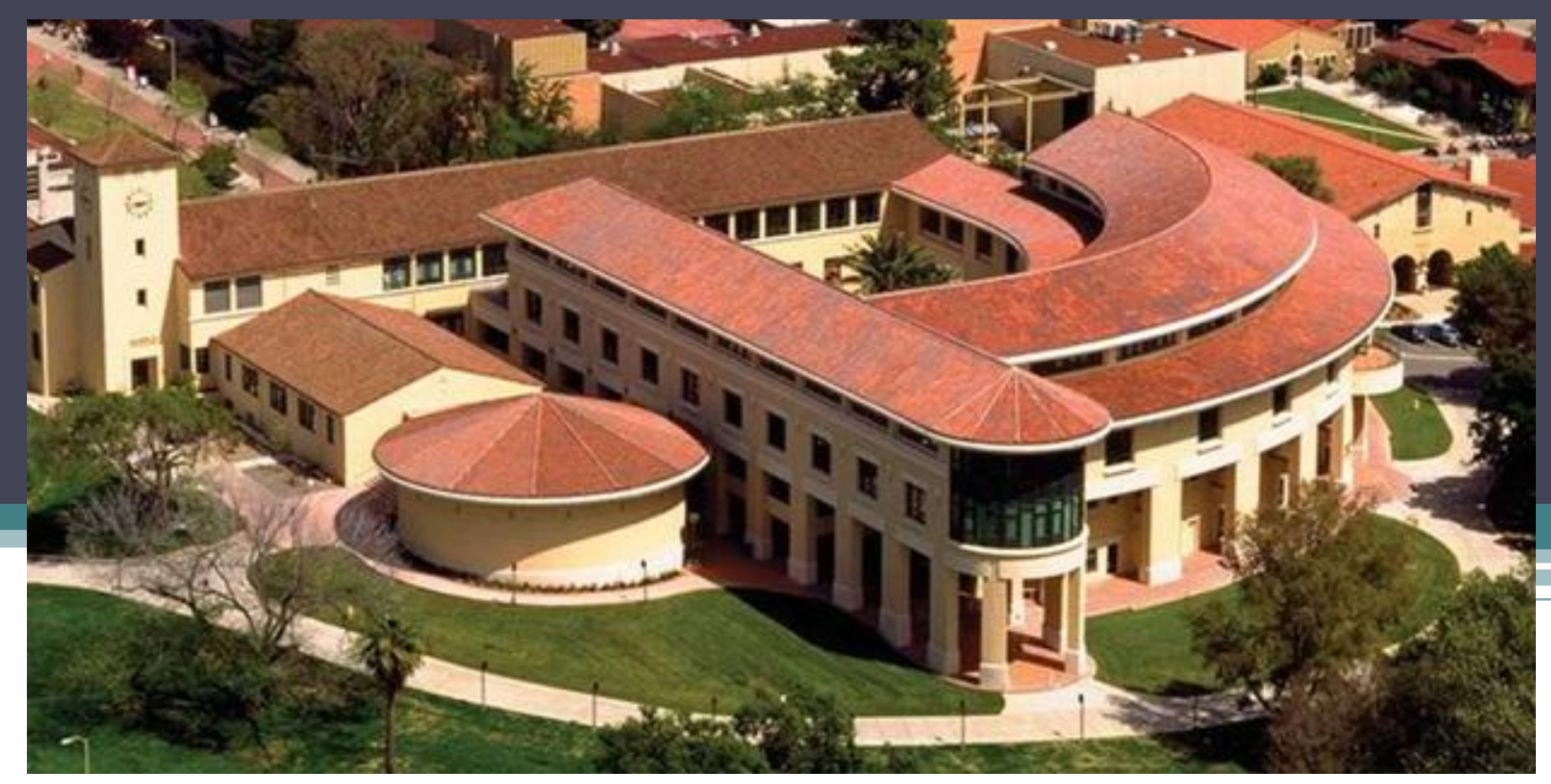

William Fletcher

Fire Protection Engineering Department

Cal Poly San Luis Obispo

Culminating Experience Presentation

March 12, 2012 


\section{Outline}

- Overview of the Building

- Fire Protection Systems
- Egress
- Fire Alarm and Detection
- Water Based Suppression
- Structural Fire Protection

- Potential Hazards and Non-Conforming Conditions

- Performance Analysis

- Results

- Recommendations 


\section{Overview of the Building}

- Completed in 1992

- Increased useable space by $73,738 \mathrm{ft}^{2}$

- Cost of $\$ 13.5$ million

- The building was designed and constructed without Automatic Fire Suppression

- Type II FR UBC 1985

- Type II A CBC 2010

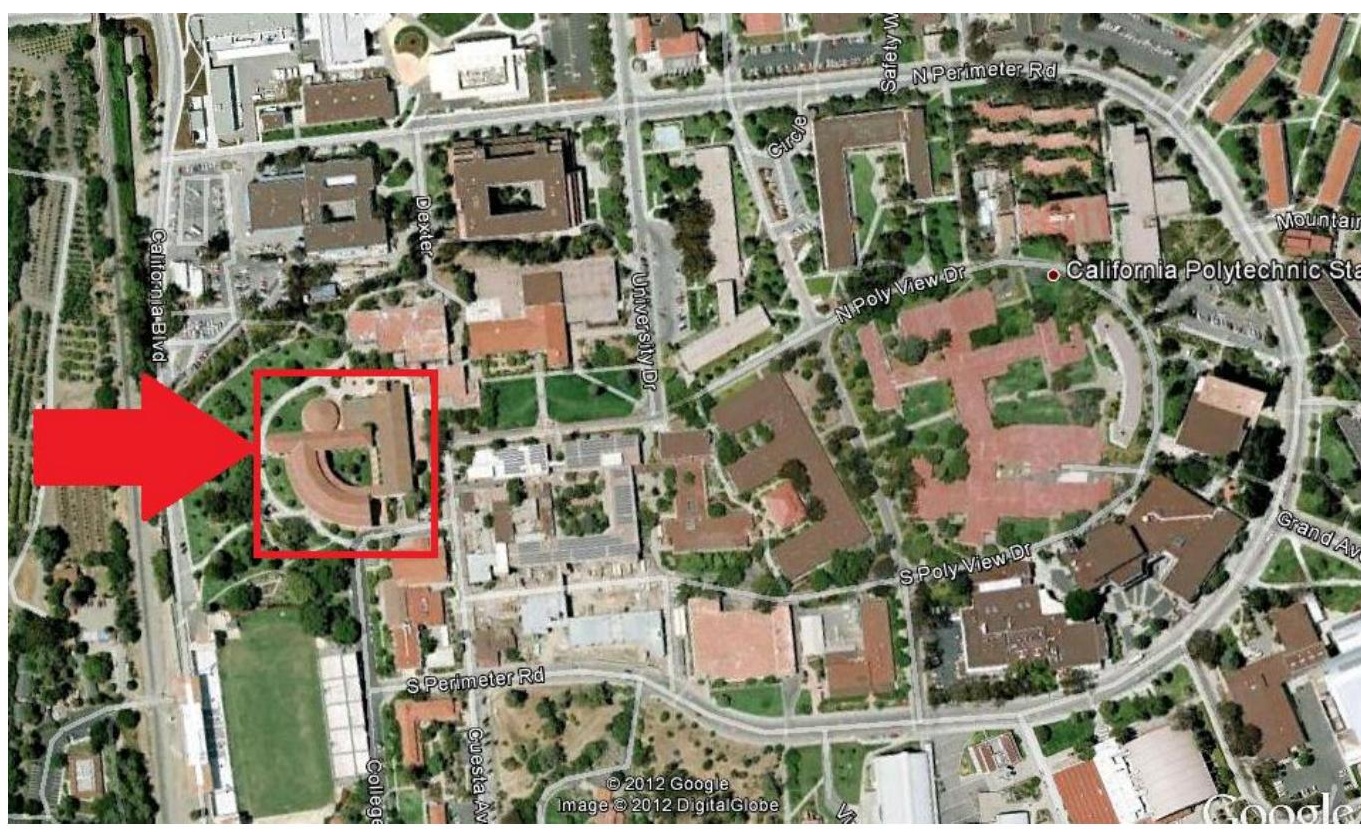




\section{Building Description}

- Occupancy Classification:

- Life Safety Code

- Existing Business Building

- Assembly

- California Building Code

- Business

- A-3

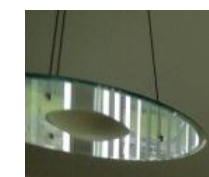

- The uses and room types include:

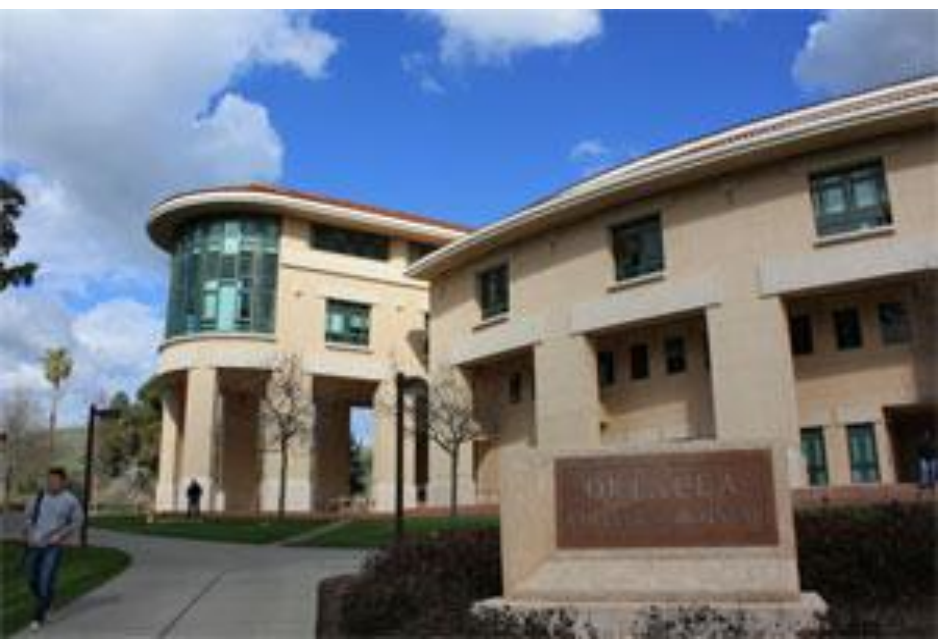

- Lecture Halls

- Classrooms (<50 persons)

- Administrative Offices

- Faculty Offices

- Conference Rooms

- Computer Labs 


\section{Business Silo}
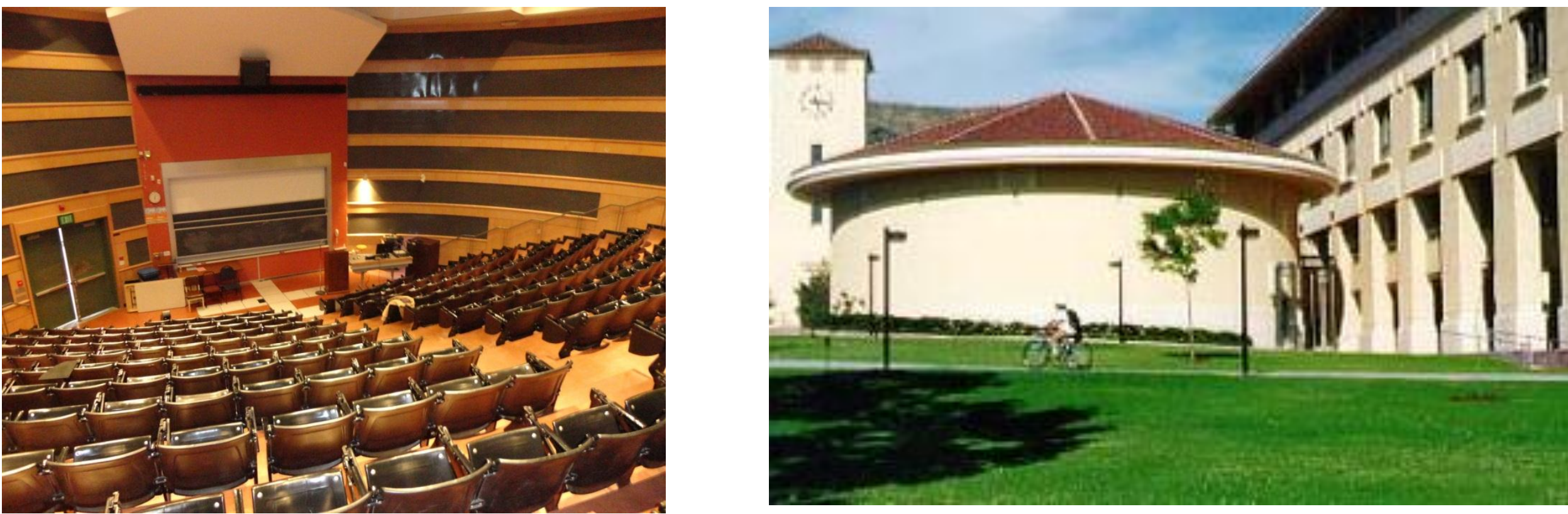

Occupant Load 240 Persons 


\section{Floor Plans: Fourth Floor}

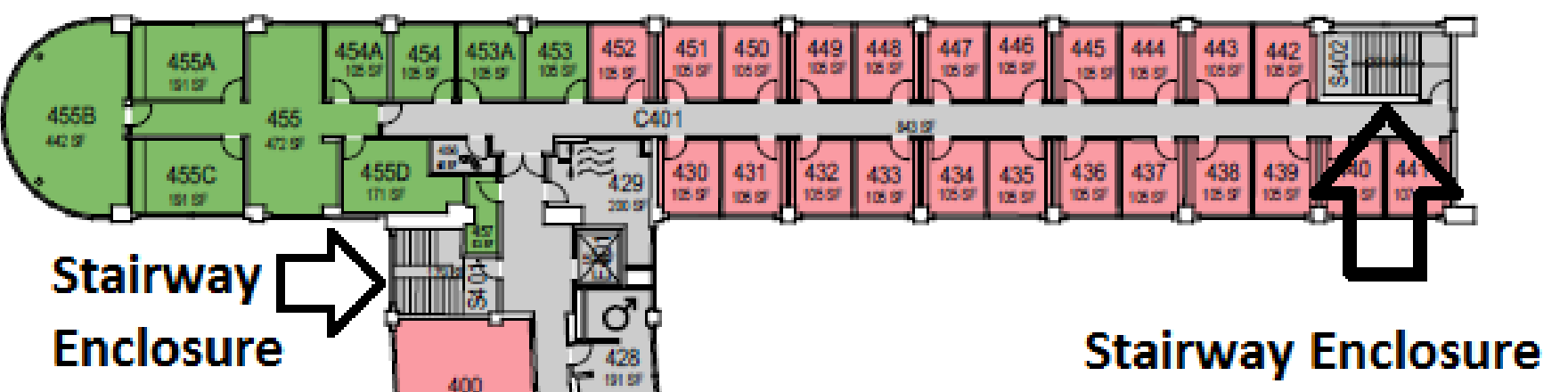

- Occupant Load: 130 Persons

- Exit Capacity: 480 Persons

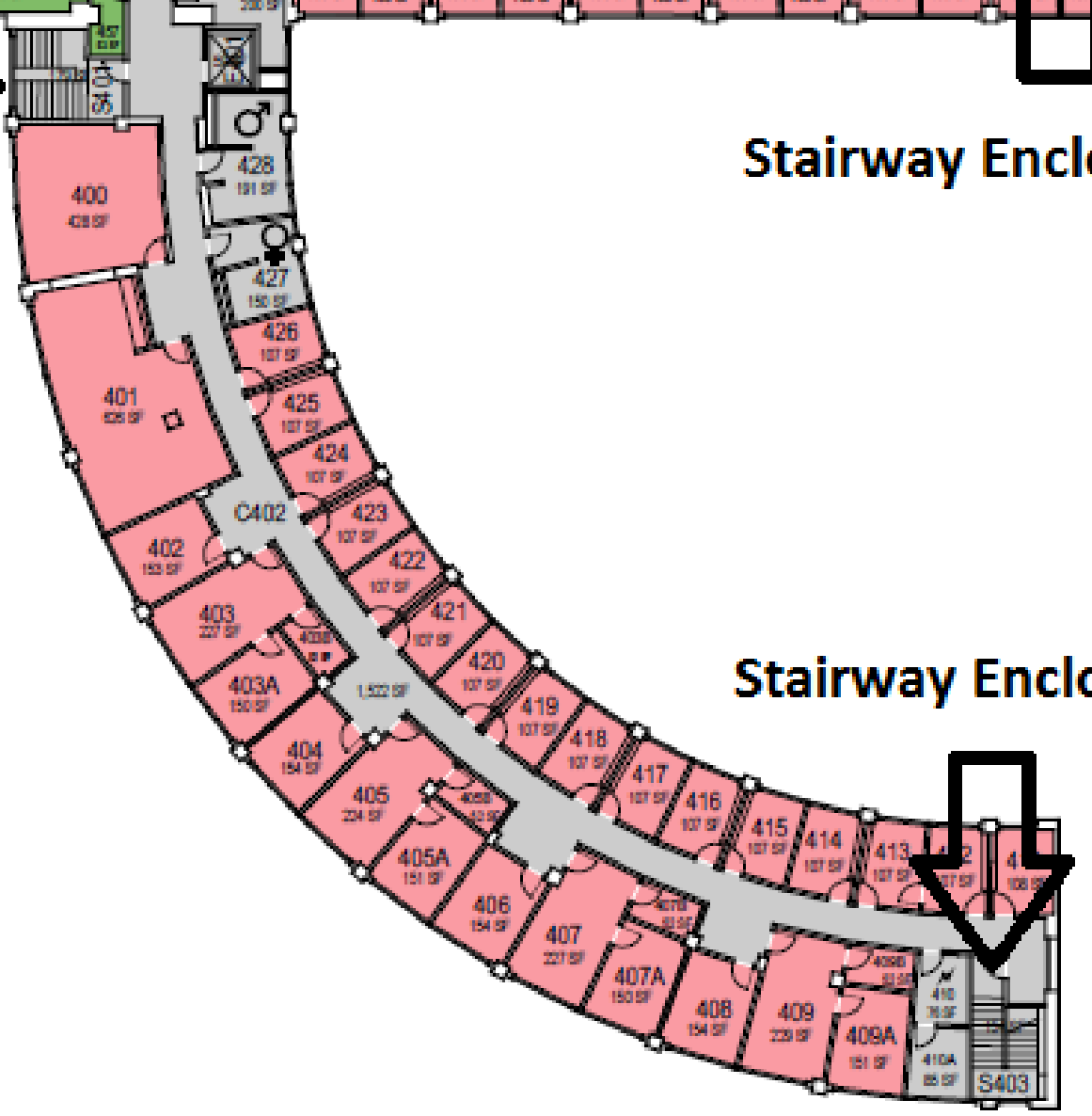




\section{Floor Plans: Third Floor}

- Occupant Load: 447 Persons

- Exit Capacity: 510 Persons

Discharge From Fourth Floor

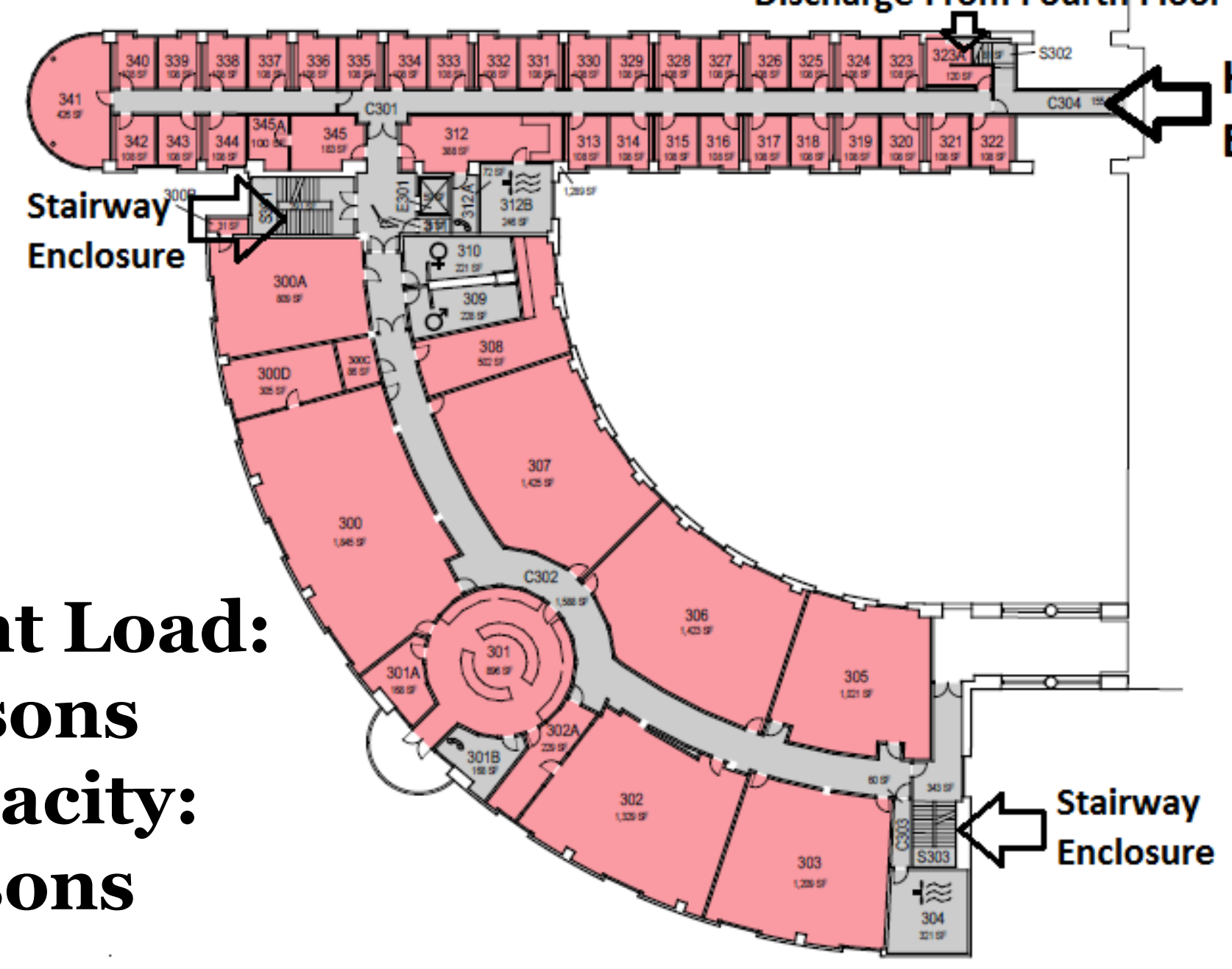

Horizontal Exit 


\section{Floor Plans:}

\section{Second Floor}

Business Silo

- Occupant Load: 240 Persons

- Exit Capacity: 360 Persons

\section{Main Building}

- Occupant Load: 504 Persons

- Exit Capacity: 680 Persons

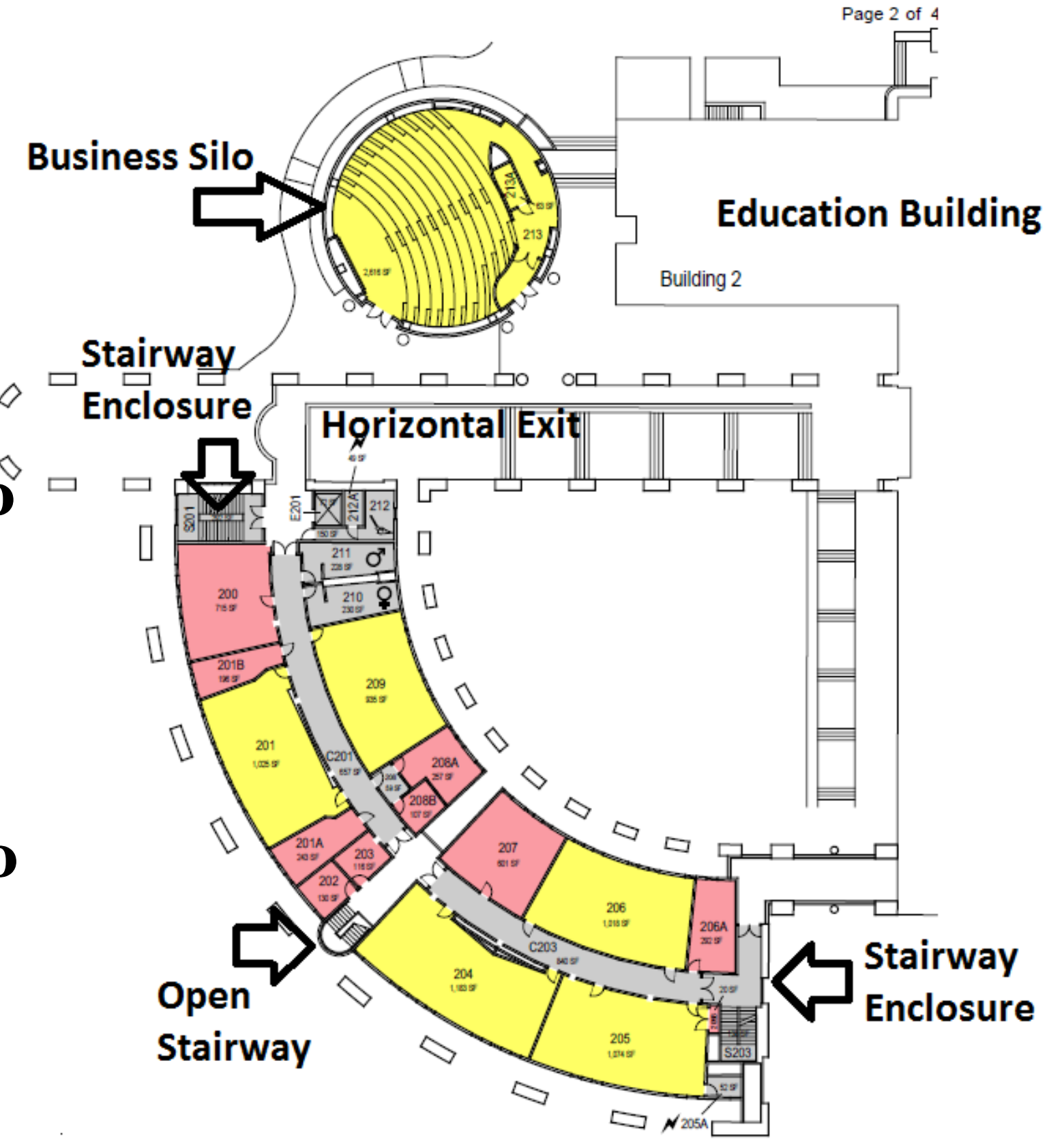




\section{Floor Plans: First Floor}

- Occupant Load:

377 Persons

- All Rooms

Open to

Outside with

Access to

Public Way

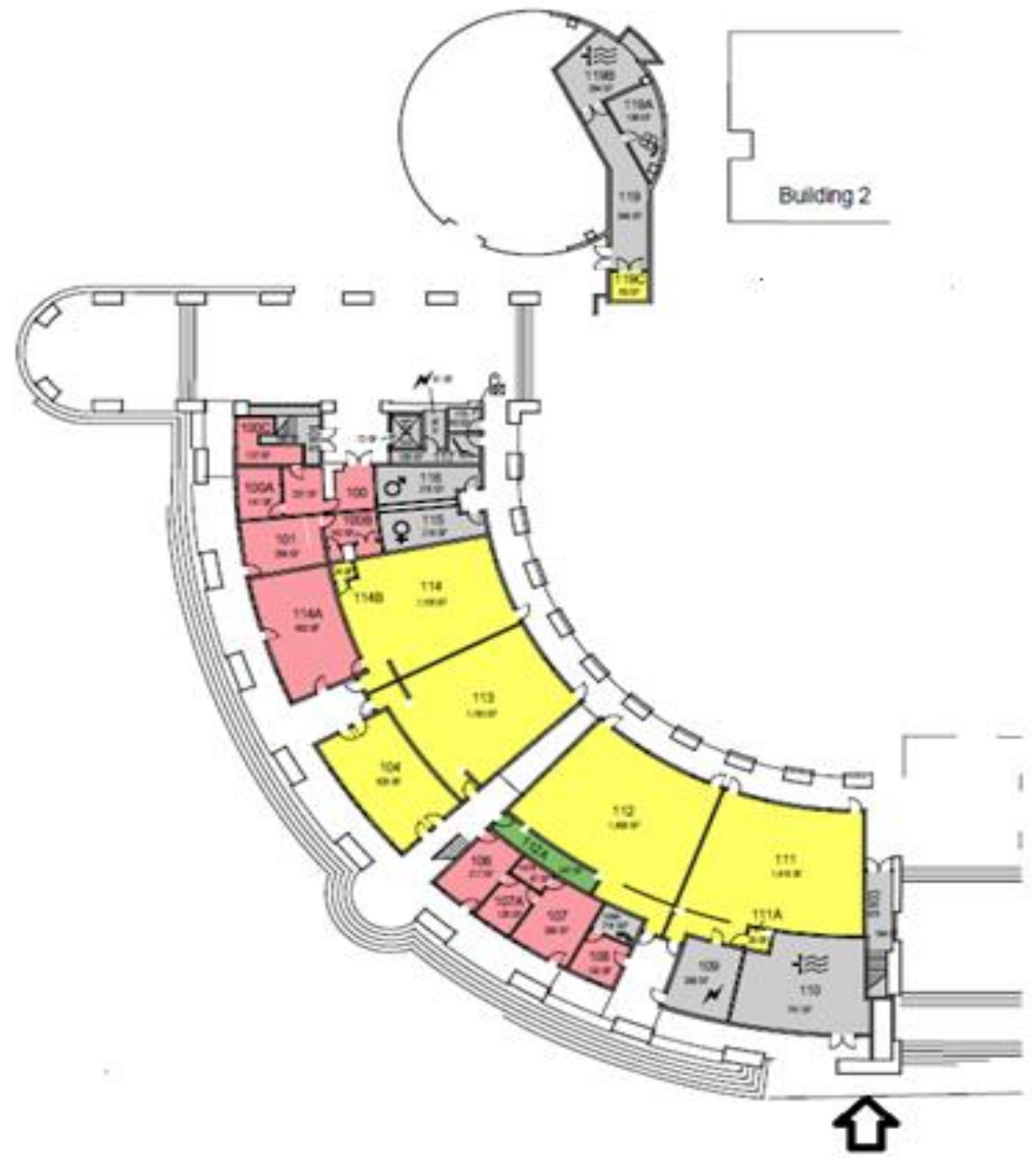

Fire Department Connections Wet-Pipe and Dry-Pipe 


\section{Exit Arrangement}

- Common Path Limits:

- Two rooms exceed the allowable of 20 feet with distance of 35 feet

- Dead-End Limits:

- Are met through limiting the distance to 50 feet with a fire door

- Travel Distance Limits:

- All below allowable 200 feet 


\section{Fire Alarm and Detection}

- The fire alarm activates notification appliances and reports to Cal Poly's Dispatcher

- Areas of concern:

- The lower three levels have very few smoke detectors, only at the end of corridors

- Covered areas:

- Top level has the highest density of smoke detectors

- Heat detection in mechanical rooms

\begin{tabular}{|c|c|c|}
\hline Locations & Type & Number \\
\hline In Hallways & Photoelectric / Fixed Temperature & 16 \\
\hline In FACP, storage and mechanical rooms & Fixed Temperature Thermal Detector & 4 \\
\hline In circular reception area on 3rd floor & Fixed Temperature Thermal Detector & 1 \\
\hline Duct Detector & Photoelectric Sensor & 5 \\
\hline
\end{tabular}




\section{Visual Requirements}

- Current System

- Gaps in coverage

- Rooms without visual notification

\begin{tabular}{|c|c|}
\hline Floor & $\begin{array}{c}\text { Audible and } \\
\text { Visual Devices }\end{array}$ \\
\hline $\mathbf{1}$ & 5 \\
\hline $\mathbf{2}$ & 4 \\
\hline $\mathbf{3}$ & 8 \\
\hline 4 & 9 \\
\hline silo & 2 \\
\hline
\end{tabular}

Audible Requirements

- Currently system

- Meets distance requirements

- Closed doors level drops below recommended level

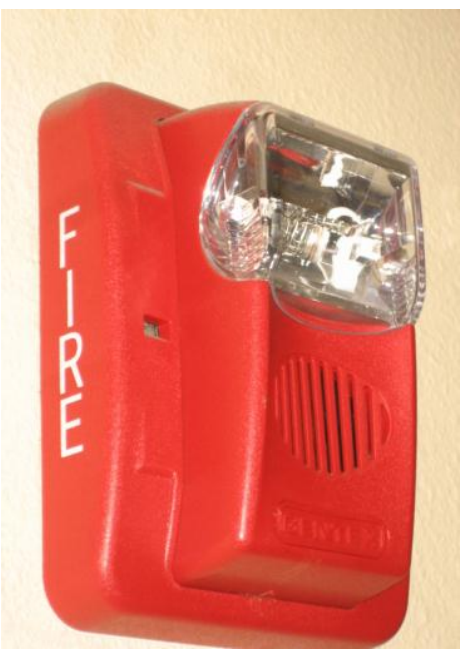




\section{Water-based Suppression}

- Dry-pipe risers in three stairwells $21 / 2$ " hook-ups

- Wet-pipe riser: fire hose cabinets with $11 / 2$ " hook-ups

- Replaced with fire extinguishers

- Fire Department Connection at south end of building 


\section{Structural Fire Protection}

- UBC Type II Fire Resistive

- CBC Type IIA

- Non-Sprinklered

- Non-rated openings and operable windows

- Exterior bearing walls only 1 hour

\begin{tabular}{|c|c|c|}
\hline Element & CBC 2010 Requirements & Construction status \\
\hline Primary Structural frame & 1 & 2 \\
\hline Bearing walls Exterior & 1 & 1 \\
\hline Bearing Walls Interior & 1 & - \\
\hline $\begin{array}{c}\text { Non-bearing walls and partitions } \\
\text { interior }\end{array}$ & 0 & 0 \\
\hline $\begin{array}{c}\text { Non-bearing walls and partitions } \\
\text { exterior with separation }\end{array}$ & 0 & 0 \\
\hline $\begin{array}{c}\text { Non-bearing walls and partitions } \\
\text { exterior with no separation }\end{array}$ & 1 & 2 \\
\hline Floor and secondary & 1 & 2 \\
\hline Roof and construction & 1 & 1 \\
\hline Shaft & 2 & 2 \\
\hline Corridor & 1 & 1 \\
\hline
\end{tabular}

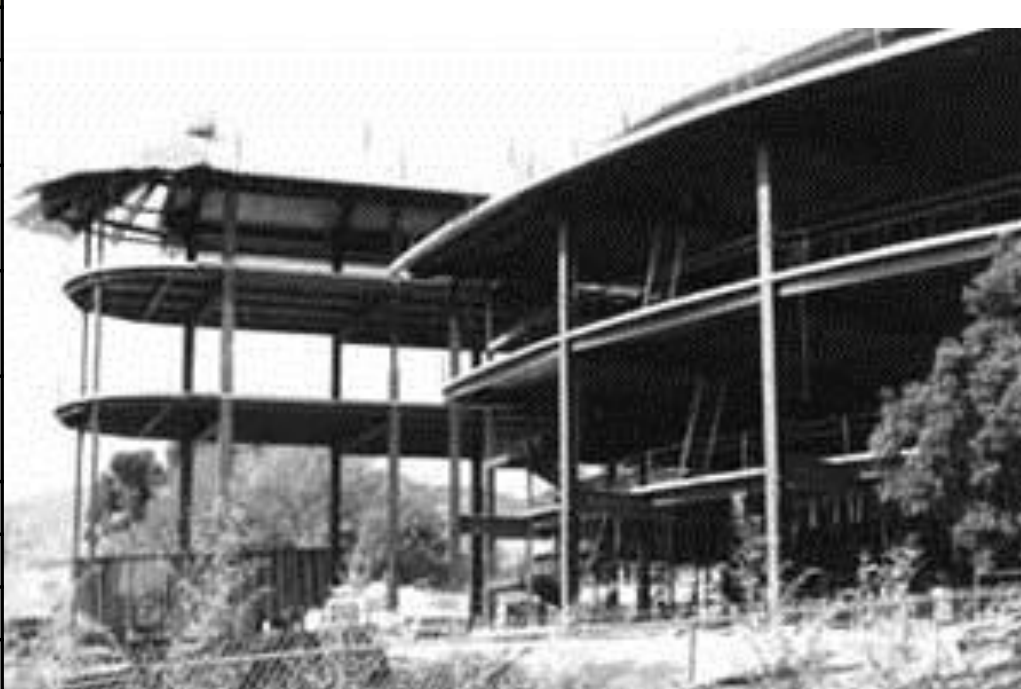




\section{Fire Resistance Ratings}

\section{Fourth Floor}

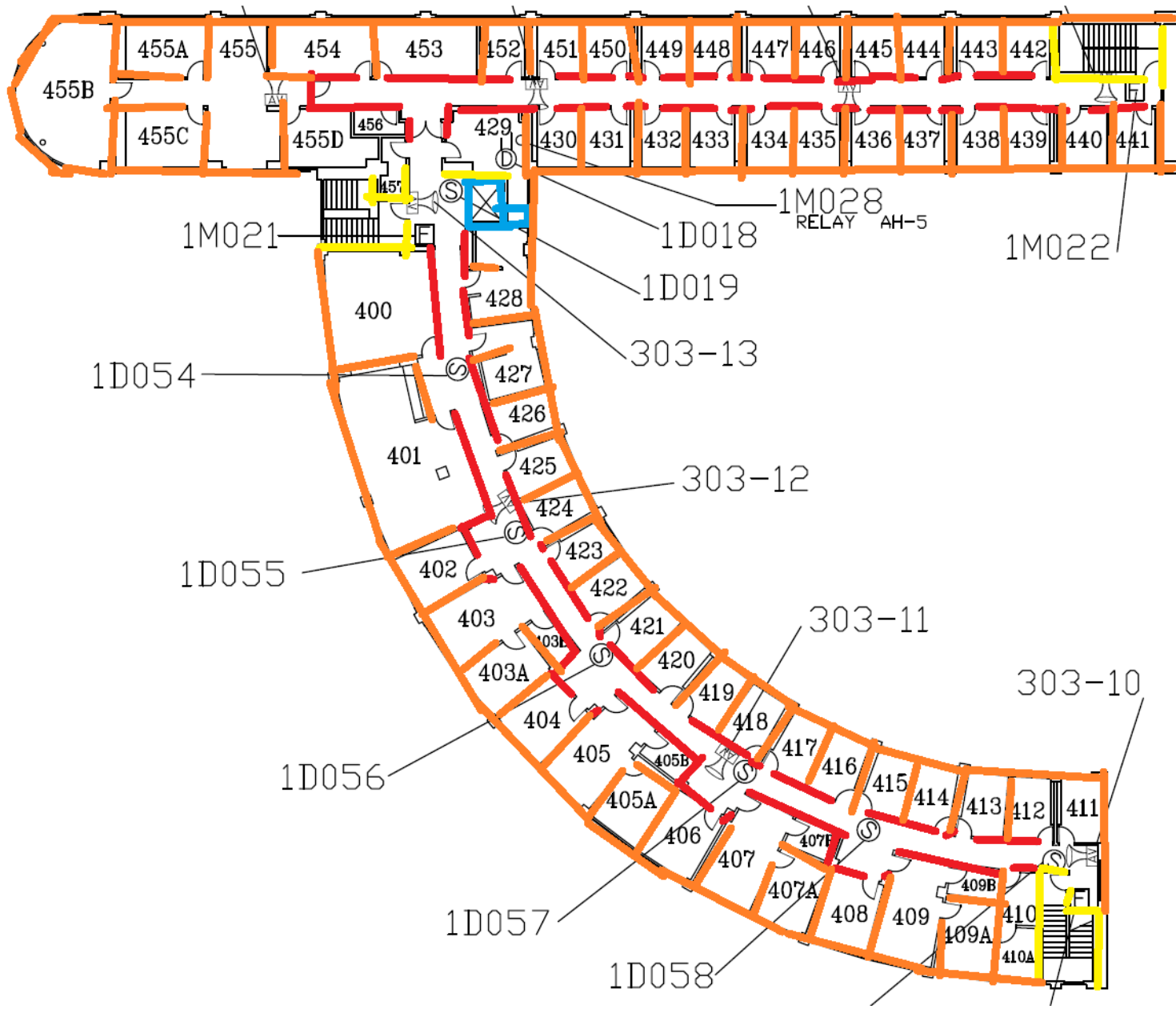

\begin{tabular}{|c|c|}
\hline \multicolumn{2}{|c|}{ Fire Resistance Key } \\
\hline & Non-Combustible \\
\hline & 1- hr. Rated GWB Partition \\
\hline & 2- hr Rated GWB Partition \\
\hline 2- hr Rated GWB Shaft wall \\
\hline Acoustic Isolated Double Stud Wall \\
\hline
\end{tabular}


Potential Hazards and

Non-Conforming Conditions

- Exit Sign Locations

- Exterior Exposure

- Passive Fire Protection

- Detector Location

- Combustibles and Obstructions in Exit Corridor 


\section{Exit Sign Locations}

- Exit Sign Locations

- Fourth Floor

- View from Fire Scenario 1

- LSC 7.10.2 Directional Signs
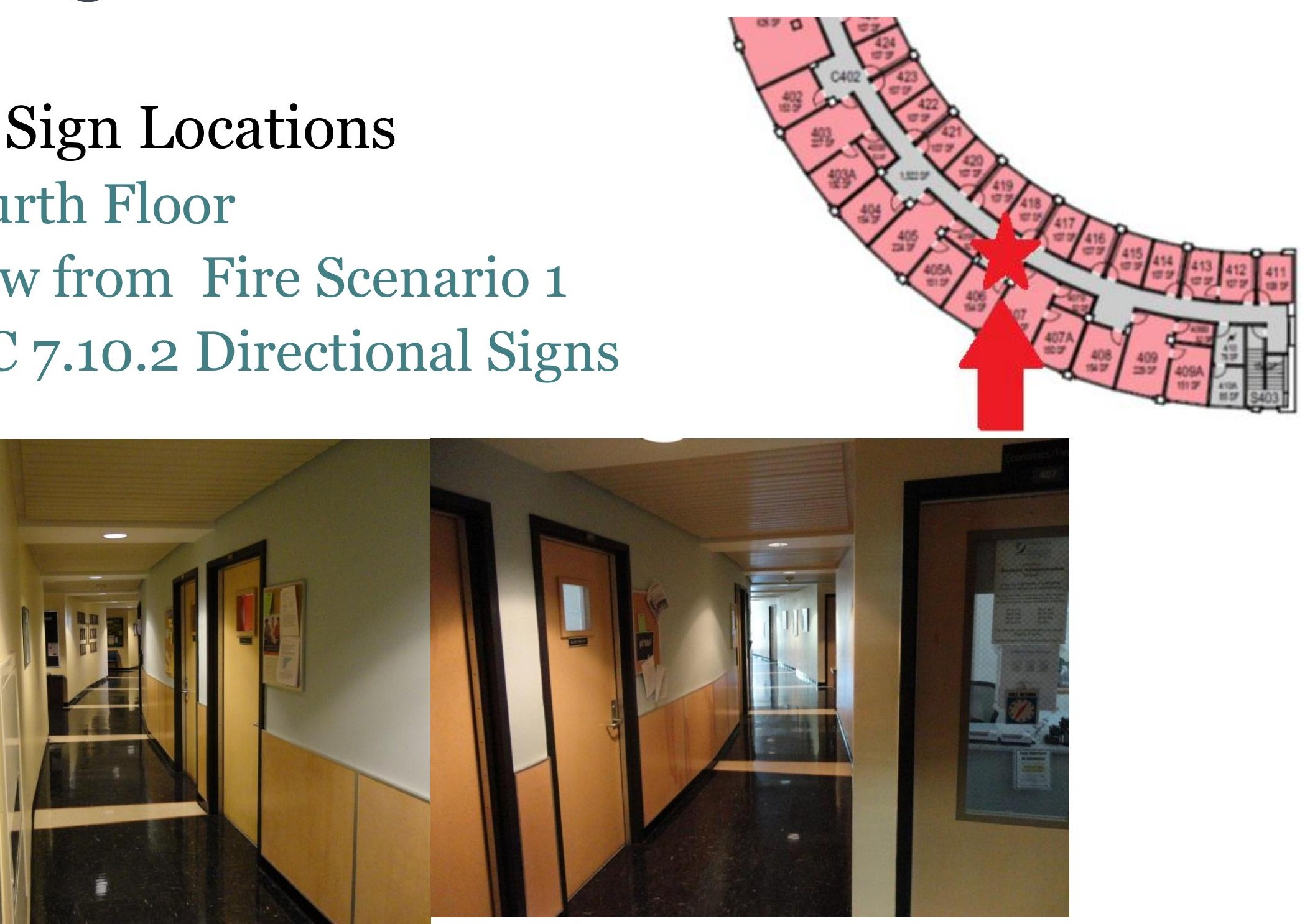


\section{Exit Sign Locations}

- Exit Sign Locations

- Third Floor

- Both Doors Propped Open

- LSC 7.10.1.2.2 Horizontal components
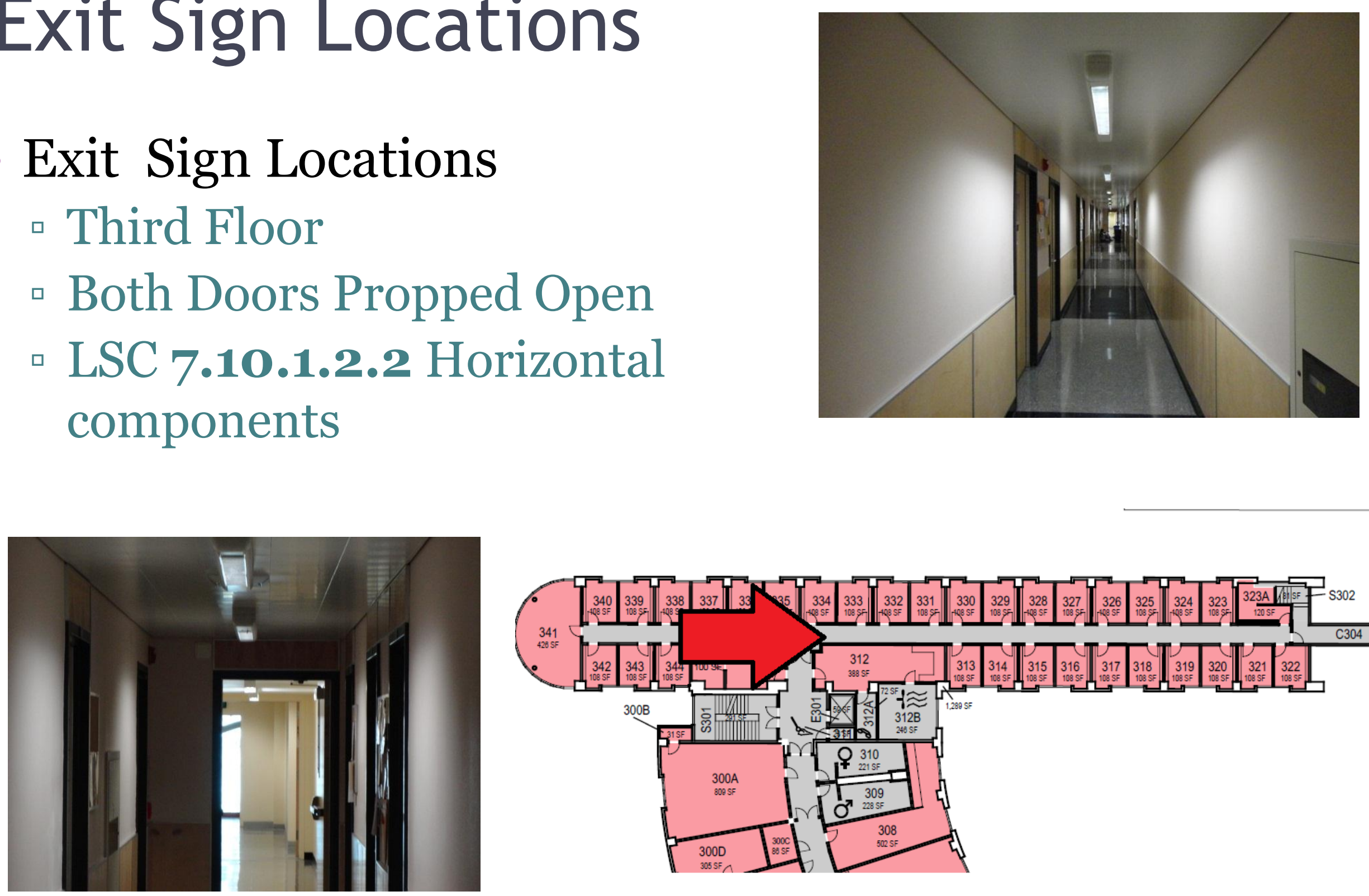


\section{Exterior Exposure}

- Exterior Exposure

- No Ratings on Operable Windows

- 20 Feet of Separation

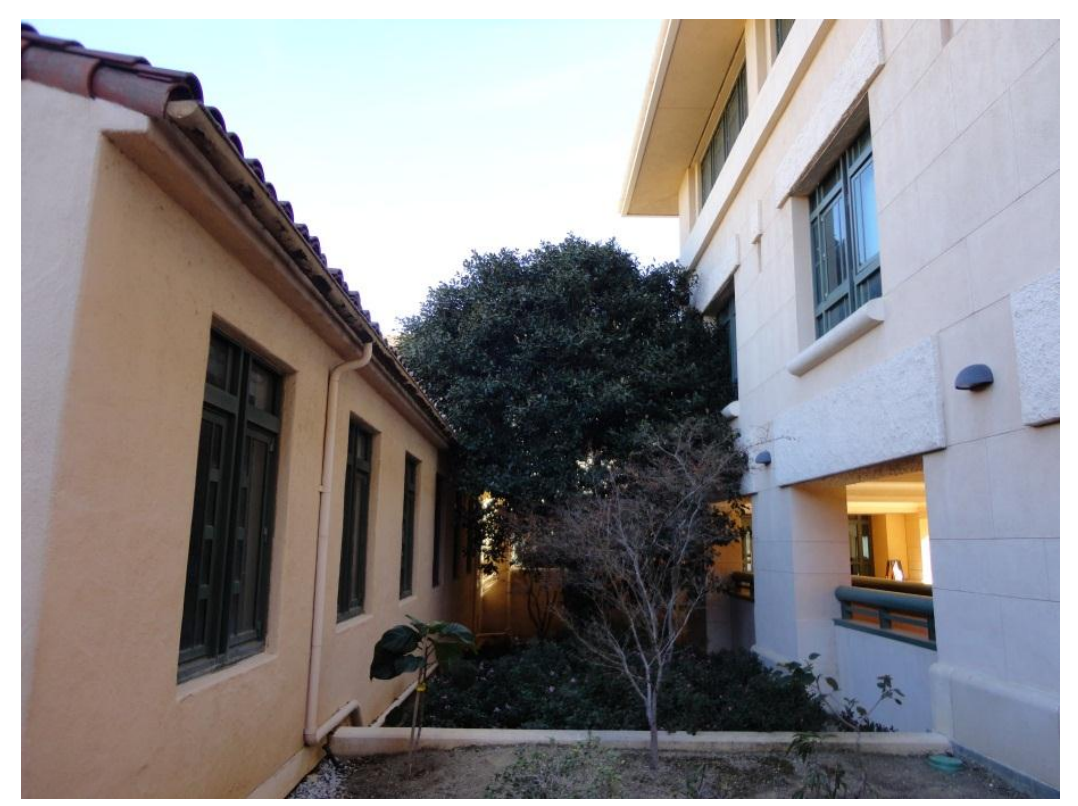




\section{Passive Fire Protection \\ - Fire Doors Propped Open}

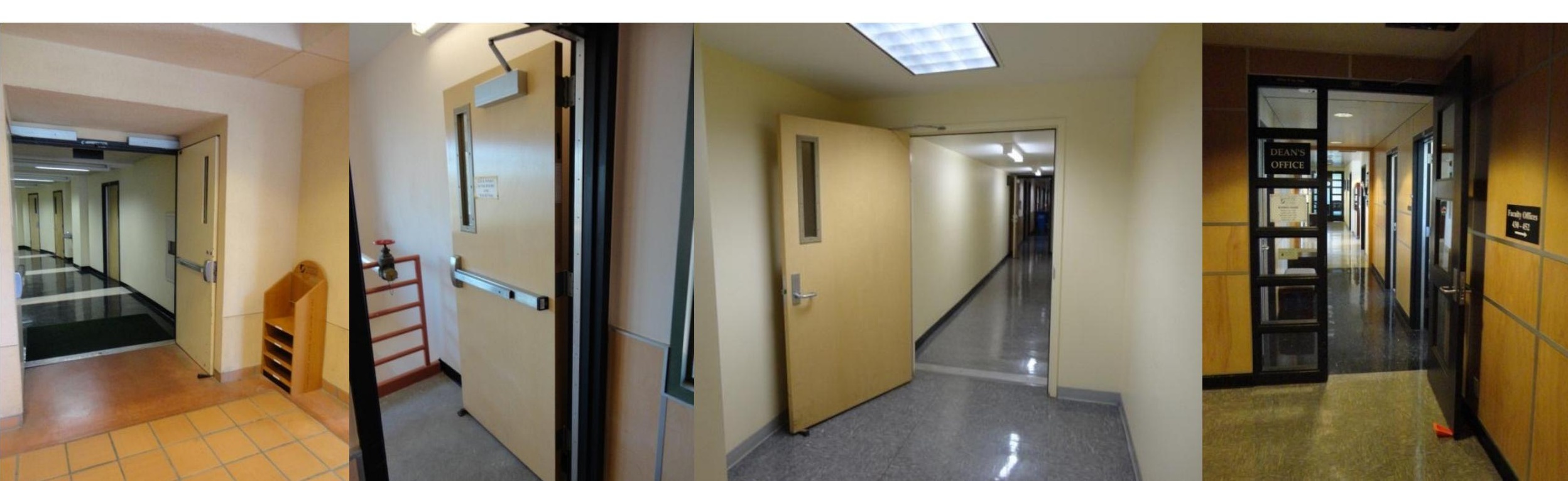




\section{Combustibles and Obstructions in}

\section{Exit Corridor}

- Corridor Width with Obstruction 39"

- Required Width 44"
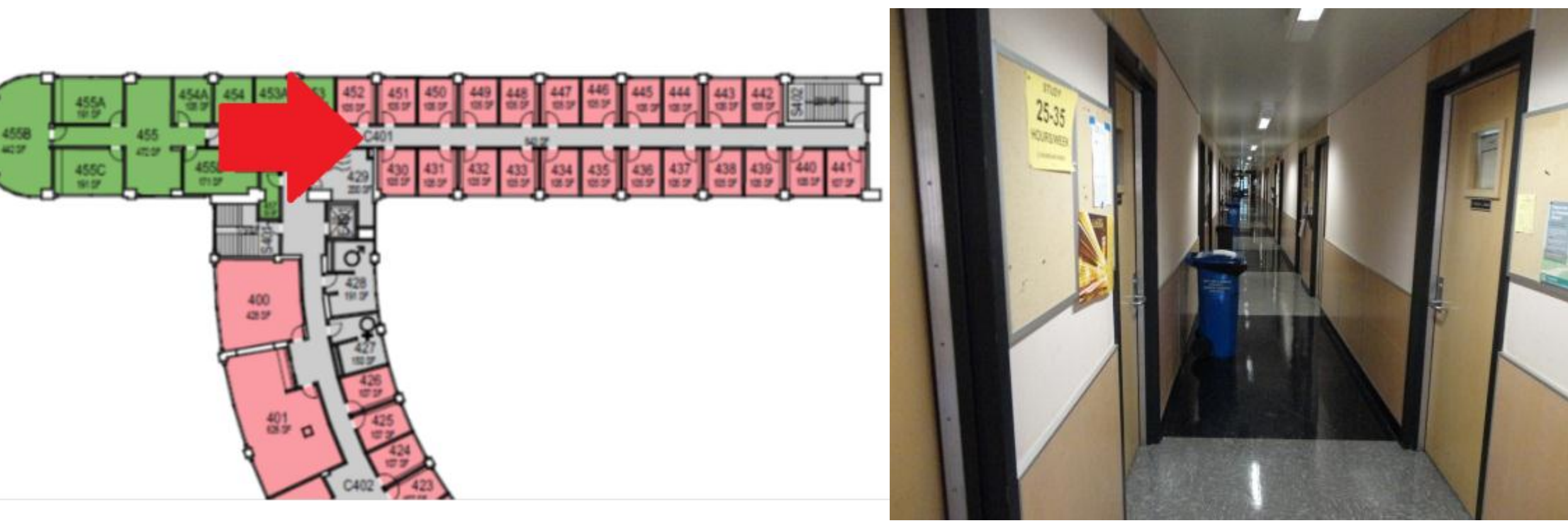


\section{Performance-Based Analysis}

- Performance Criteria

- Fire Dynamics Simulator

- Fire Scenarios

- Scenario 1

- Scenario 2

- Scenario 3

- Egress Analysis

- Required Safe Egress Time(RSET)

- Results 


\begin{tabular}{|c|c|c|}
\hline Fire Protection Goals & Objectives & Performance Criteria \\
\hline \multirow{6}{*}{$\begin{array}{l}\text { Minimize fire-related injuries } \\
\text { and prevent undue loss of life }\end{array}$} & \multirow{4}{*}{$\begin{array}{l}\text { Maintain tenable } \\
\text { conditions in } \\
\text { paths of egress }\end{array}$} & $\mathrm{CO}<1,400 \mathrm{ppm}$ \\
\hline & & Temperature $<60 \mathrm{C}$ \\
\hline & & \begin{tabular}{|c|} 
Visibility greater than 20 feet or \\
Optical density greater than 0.2 \\
$1 / \mathrm{m}$ \\
\end{tabular} \\
\hline & & Smoke level 6 feet above floor \\
\hline & \multirow{2}{*}{$\begin{array}{c}\text { Prevent flash } \\
\text { over in room of } \\
\text { origin }\end{array}$} & $\begin{array}{c}\text { Temperature inside of room and } \\
\text { upper layer }<500 \mathrm{C}\end{array}$ \\
\hline & & $\mathrm{q}^{\prime \prime r}$ - floor level $<20 \mathrm{~kW} / \mathrm{m}^{\wedge} 2$ \\
\hline
\end{tabular}

Sources: The SFPE Handbook: Bryan, J.L., "Behavioral Response to Fire and Smoke," Purser, D.A., "Toxicity Assessment of Combustion Products," "Assessment of Hazards to Occupants from Smoke, Toxic Gases and Heat”, Performance Criterion from NFPA 101 Life Safety Code. Drysdale, D. "An Introduction to Fire Dynamics.

NFPA Handbook Chapter "Combustion Products and Their Effects on Life Safety" 


\section{Fire Dynamic Simulator}

- Used as a tool to help determine Available Safe Egress Time (ASET)

- Limitations

- Based on conditions specified by user

- Fire Size, Fire location, conditions, materials, etc.

- Run times and mesh size

- Rectilinear numerical grid

- FDS will only consider CO formed directly through the combustion process

- Experimental data

- Measuring Performance Criteria in room of origin and in the means of egress 


\section{Fire Scenario: 1}

- There are open areas inside the corridor that are currently being utilized for waiting or display areas

- This places a large amount of combustibles in an exit corridor

- There are four alcoves on floor
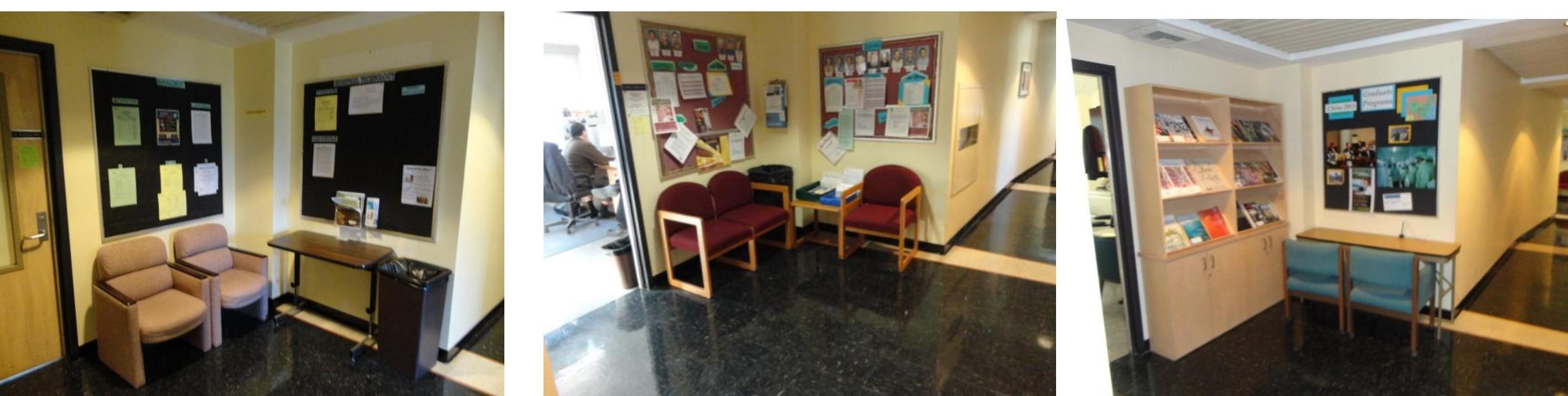


\section{Fire Scenario: 1}

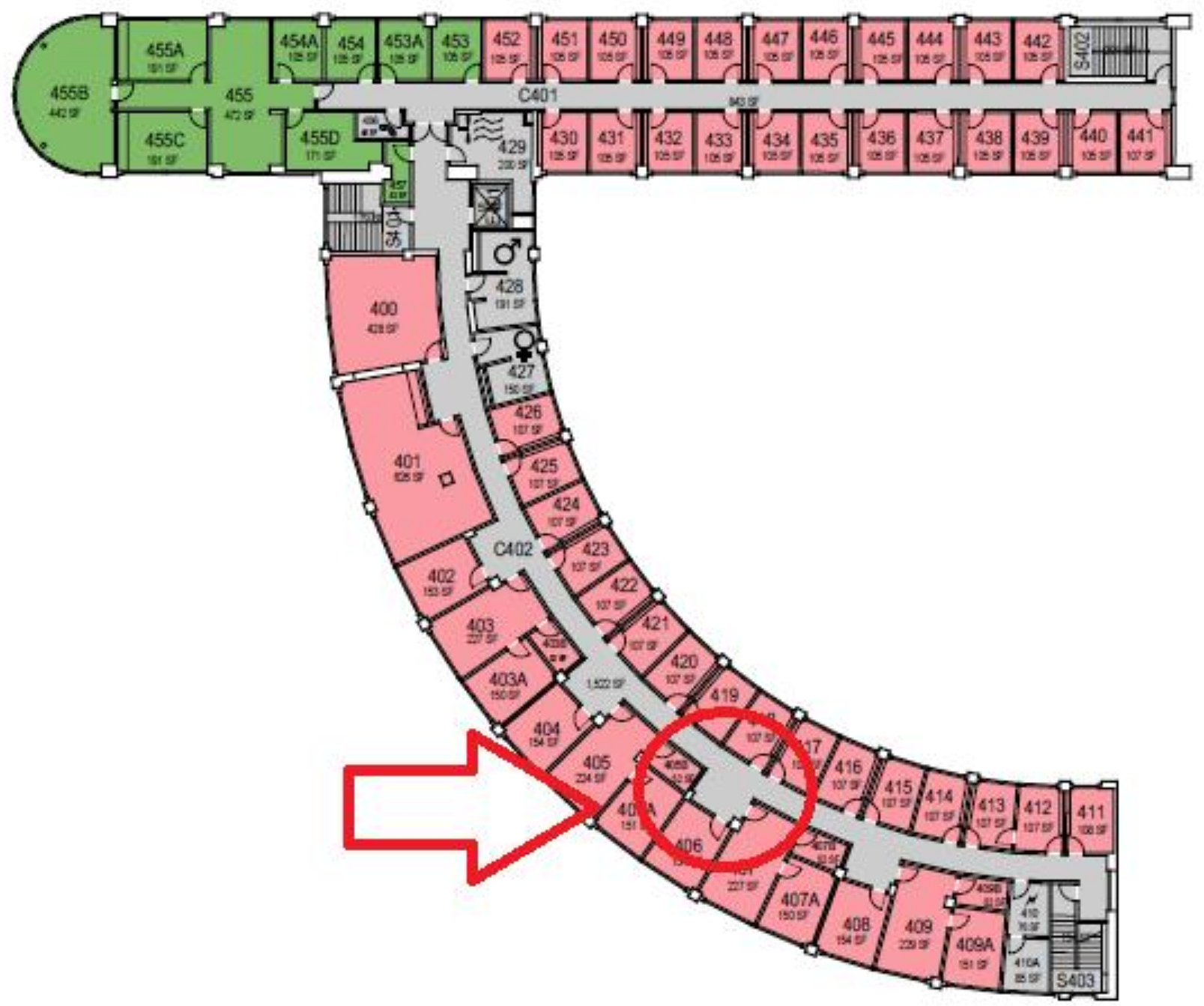




\section{Fire Scenario 1}

- Heat Release Rate

- $\mathrm{Q}=a \mathrm{t}^{2}$

- Fast: $\operatorname{tg}=150$ seconds

$\alpha=0.047$
- A peak heat release rate of $2000 \mathrm{~kW}$ was used

\section{Results of Babrauskas Studies}

\begin{tabular}{|c|c|c|c|c|}
\hline & \multicolumn{2}{|c|}{ Loveseat } & \multicolumn{2}{c|}{ Chair } \\
\hline Furniture calorimeter & $\begin{array}{c}\text { Pk HRR } \\
(\mathrm{kW})\end{array}$ & $\begin{array}{c}\text { Flashover } \\
\text { time }(\mathrm{s})\end{array}$ & $\begin{array}{c}\text { Pk HRR } \\
(\mathrm{kW})\end{array}$ & $\begin{array}{c}\text { Flashover } \\
\text { time (s) }\end{array}$ \\
\hline Room $1(2.0 \mathrm{~m} \times 1.13 \mathrm{~m})$ & 2490 & $230 *$ & 1970 & 280 * \\
\hline Room $2(2.0 \mathrm{~m} \times 1.50 \mathrm{~m})$ & 3550 & 373 & & \\
\hline Room $3(1.29 \mathrm{~m} \times 2.0 \mathrm{~m})$ & 2660 & 410 & & \\
\hline Room $4(1.29 \mathrm{~m} \times 2.0 \mathrm{~m})$ & & & 2260 & 302 \\
\hline \\
Flashover time assessed as $20 \mathrm{~kW} / \mathrm{m}^{2}$ heat flux on floor \\
\hline \\
* In furniture calorimeter, time to peak HRR is reported \\
\hline
\end{tabular}




\section{Scenario 1: ASET}

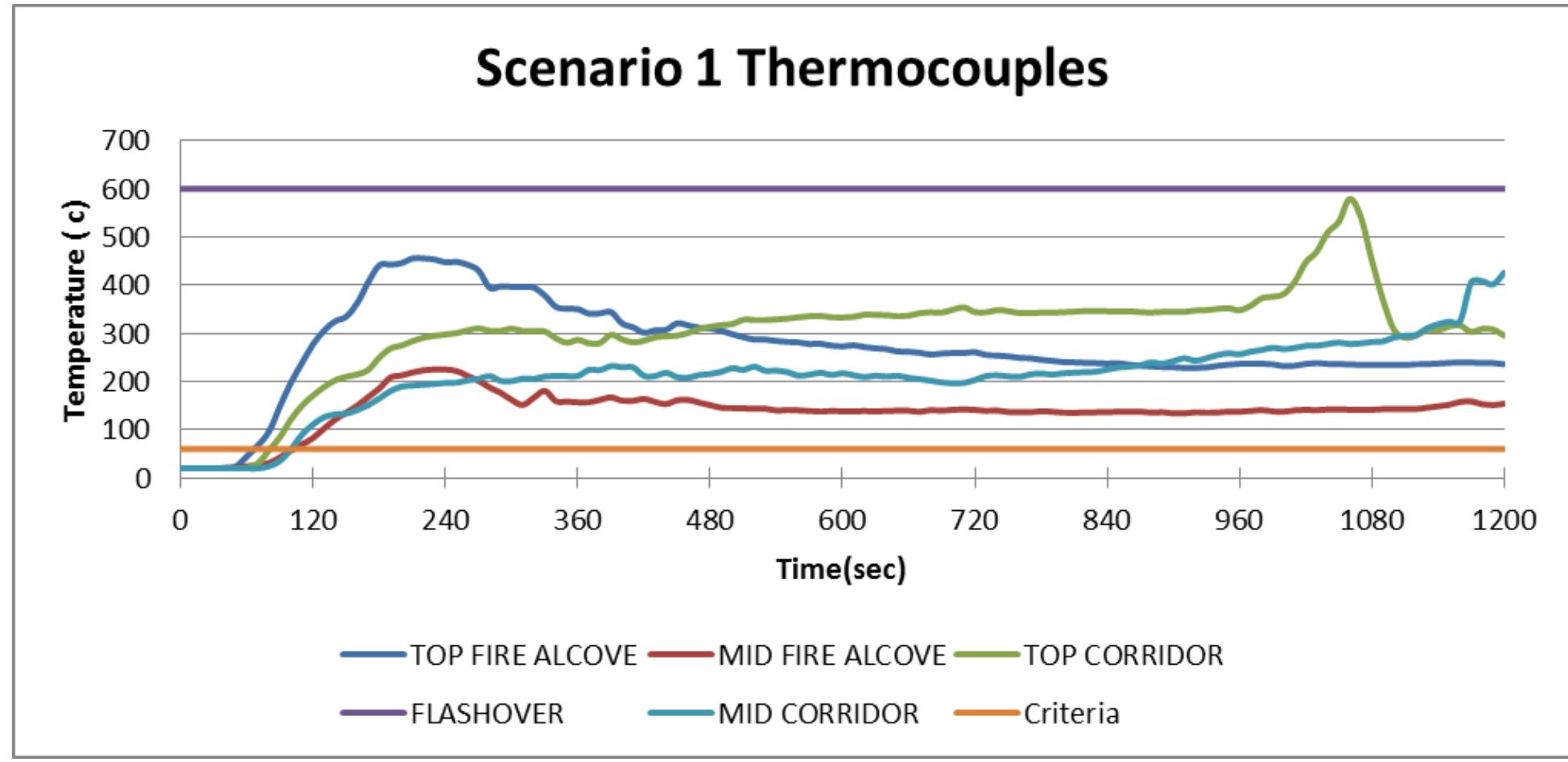

In Fire Alcove Max Temperature: $456 \mathrm{C}$ at 210 seconds In Corridor Max Temperature: $579 \mathrm{C}$ at 1060 seconds Criteria in Fire Alcove at 65 seconds and in Corridor at 82 seconds 


\section{After 120 Seconds}

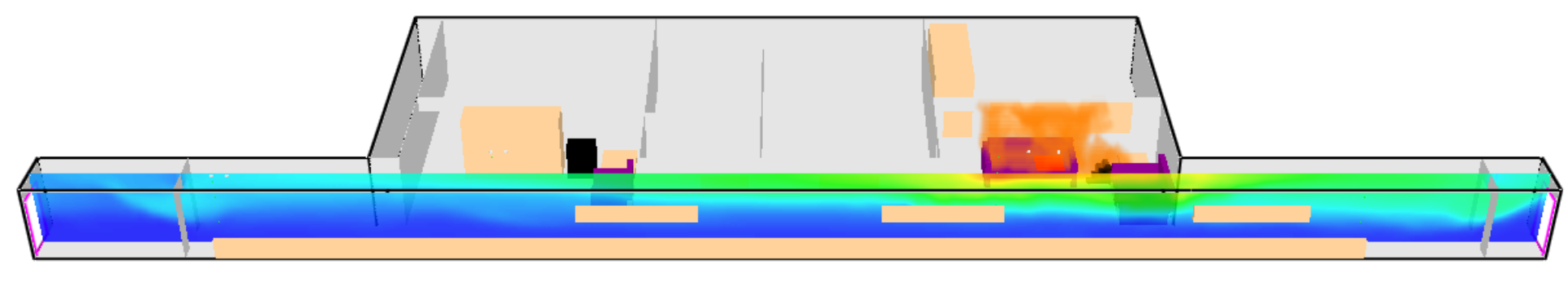

After 210 Seconds

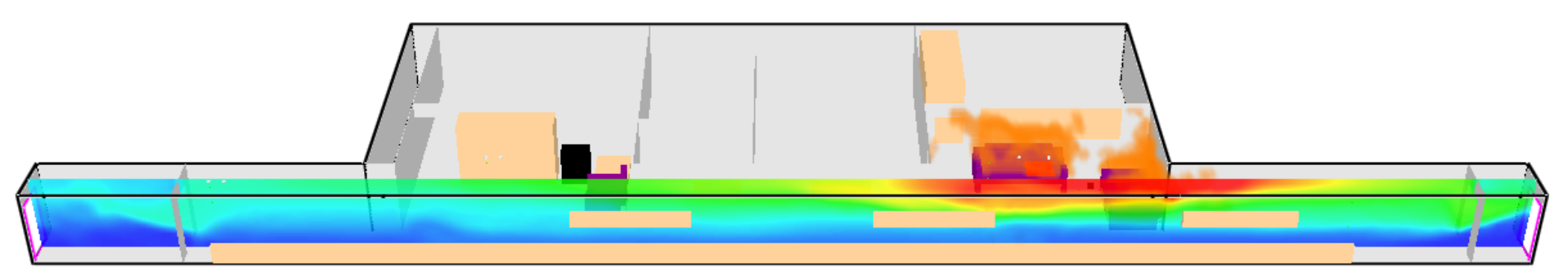




\section{Scenario 1: Heat Release Rate}

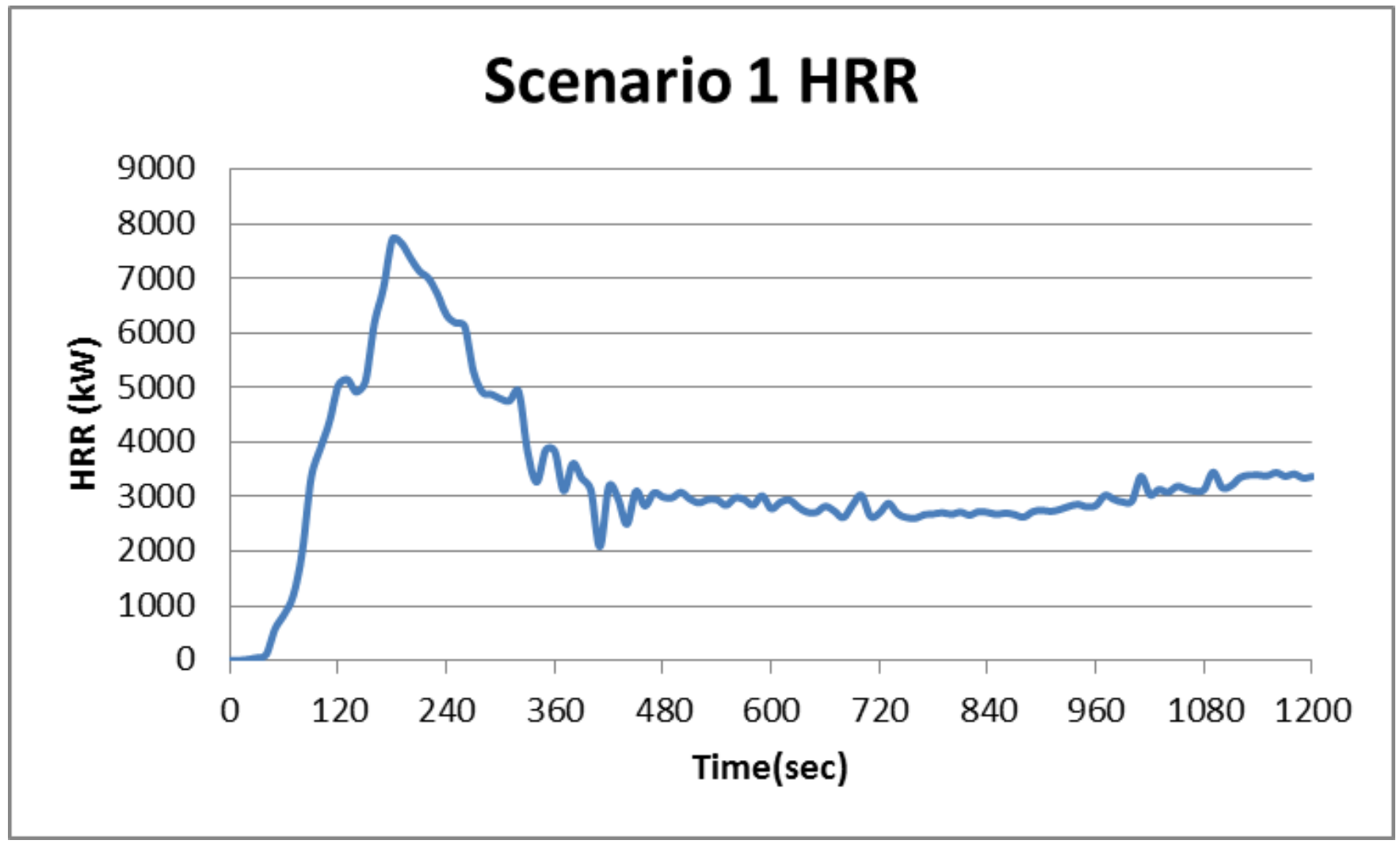

- Max Heat Release Rate: $7722 \mathrm{~kW}$ at 180 seconds 


\section{Scenario 1: Smoke Level}

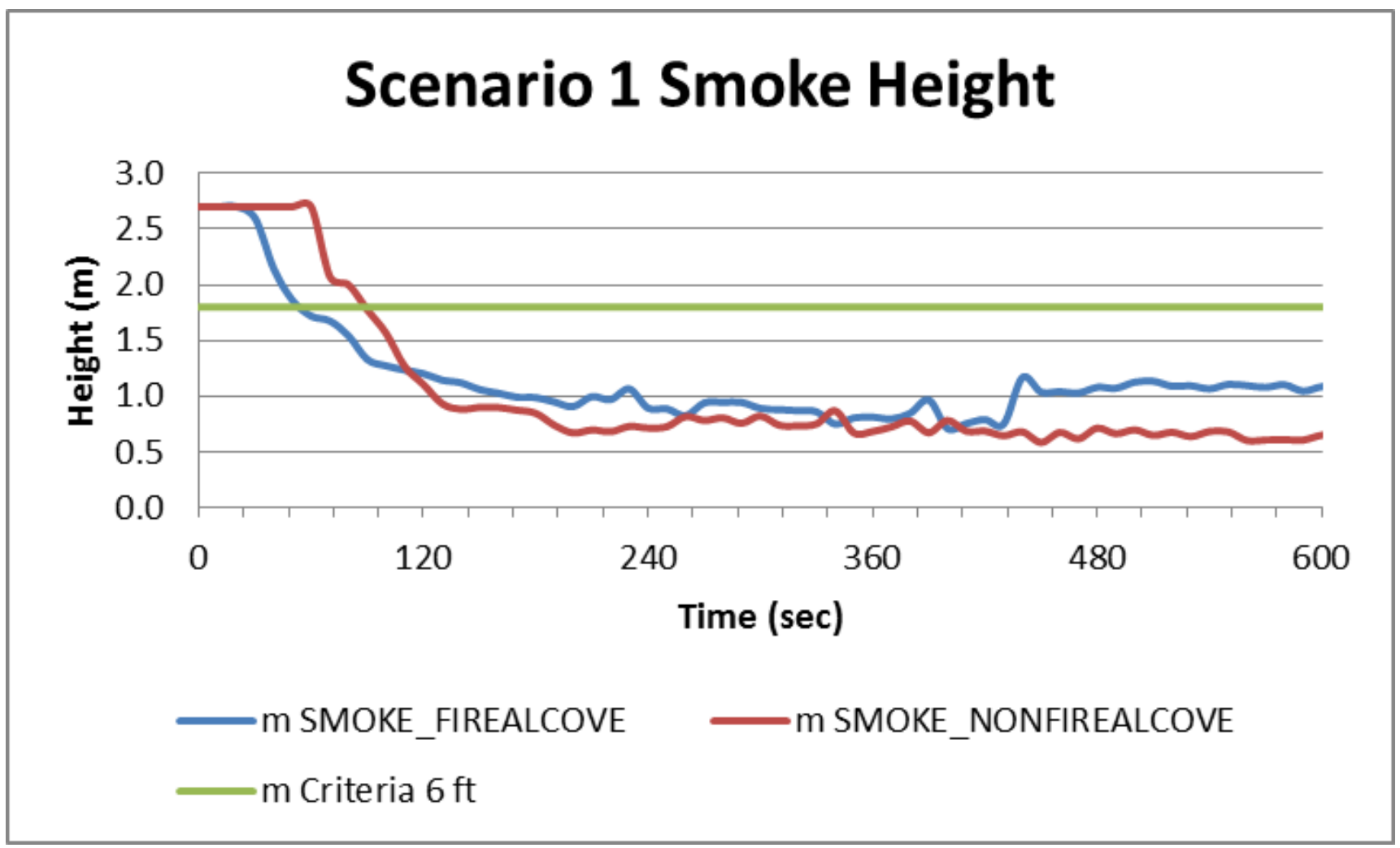

- Room at 55 seconds

- Corridor at 90 seconds 


\section{Scenario 1:Smoke Movement}

After 30 seconds

\section{After 55 seconds}

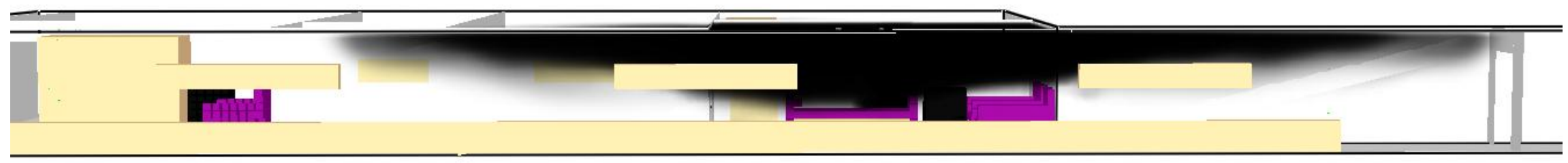

After 90 seconds

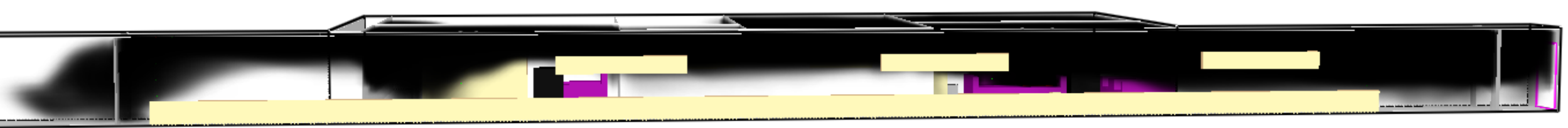




\section{Scenario 1: Visibility}

- Using Soot to approximate visibility in meters at the height of $1.5 \mathrm{~m}$

- Optical Density

- Room at 38 seconds

After 40 seconds

- Corridor at 75 seconds

After 75 seconds

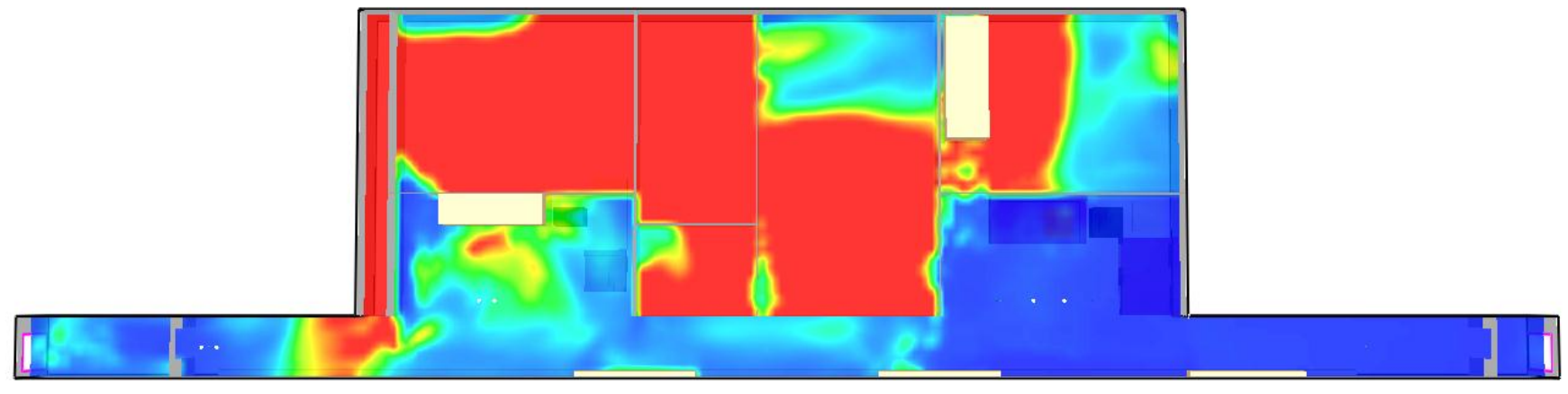




\section{Fire Scenario: 2}

- Fourth floor there is a supply room and staff break room

- Both fire doors and window are open

- Several sources of ignition

- High fuel load 


\section{Fire Scenario: 2}

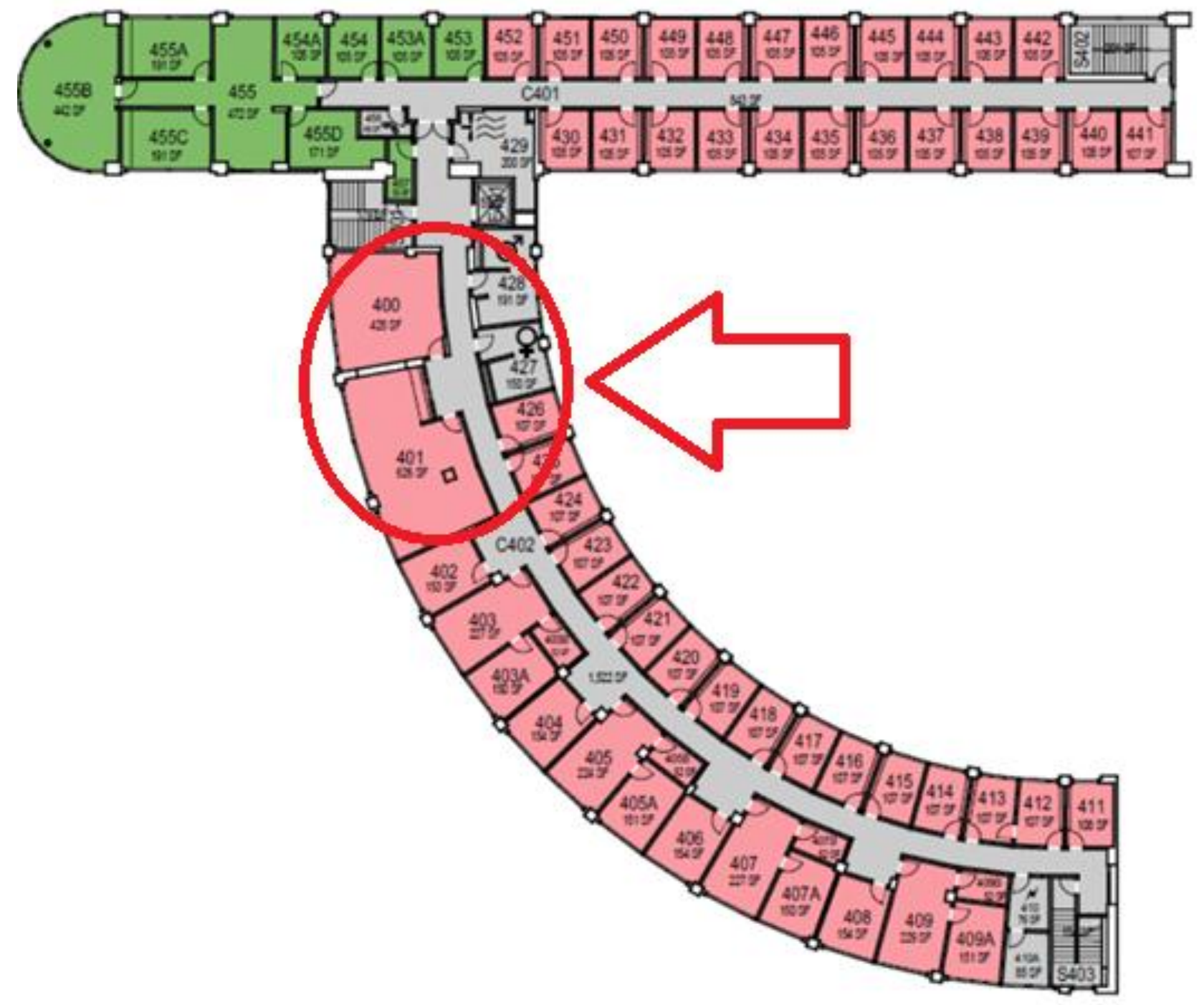




\section{Fire Scenario: 2 ASET}

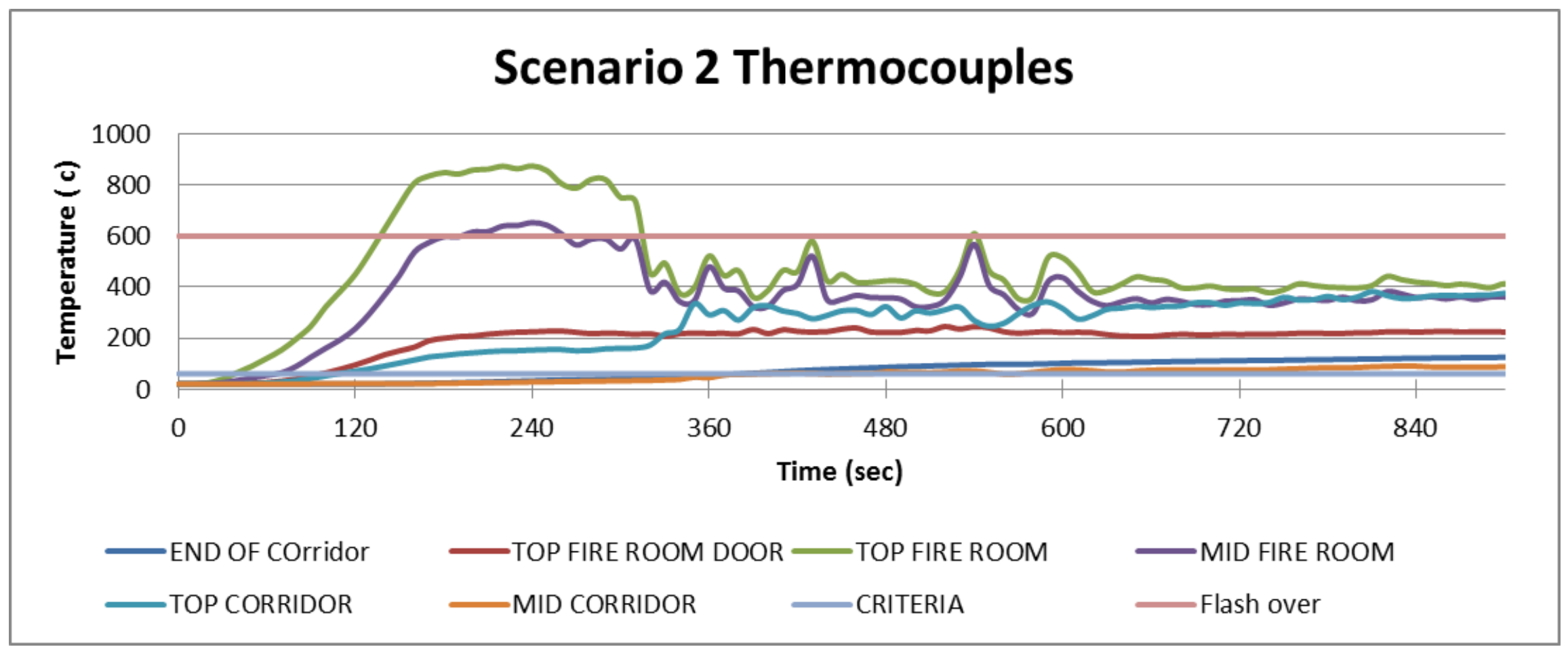

In Fire Room Max Temperature: $875 \mathrm{C}$ at 240 seconds In Corridor Max Temperature: $418 \mathrm{C}$ at 1190 seconds Criteria in Fire Room at 35 seconds and in Corridor at 105 seconds 


\section{Scenario 2: Heat Release Rate}

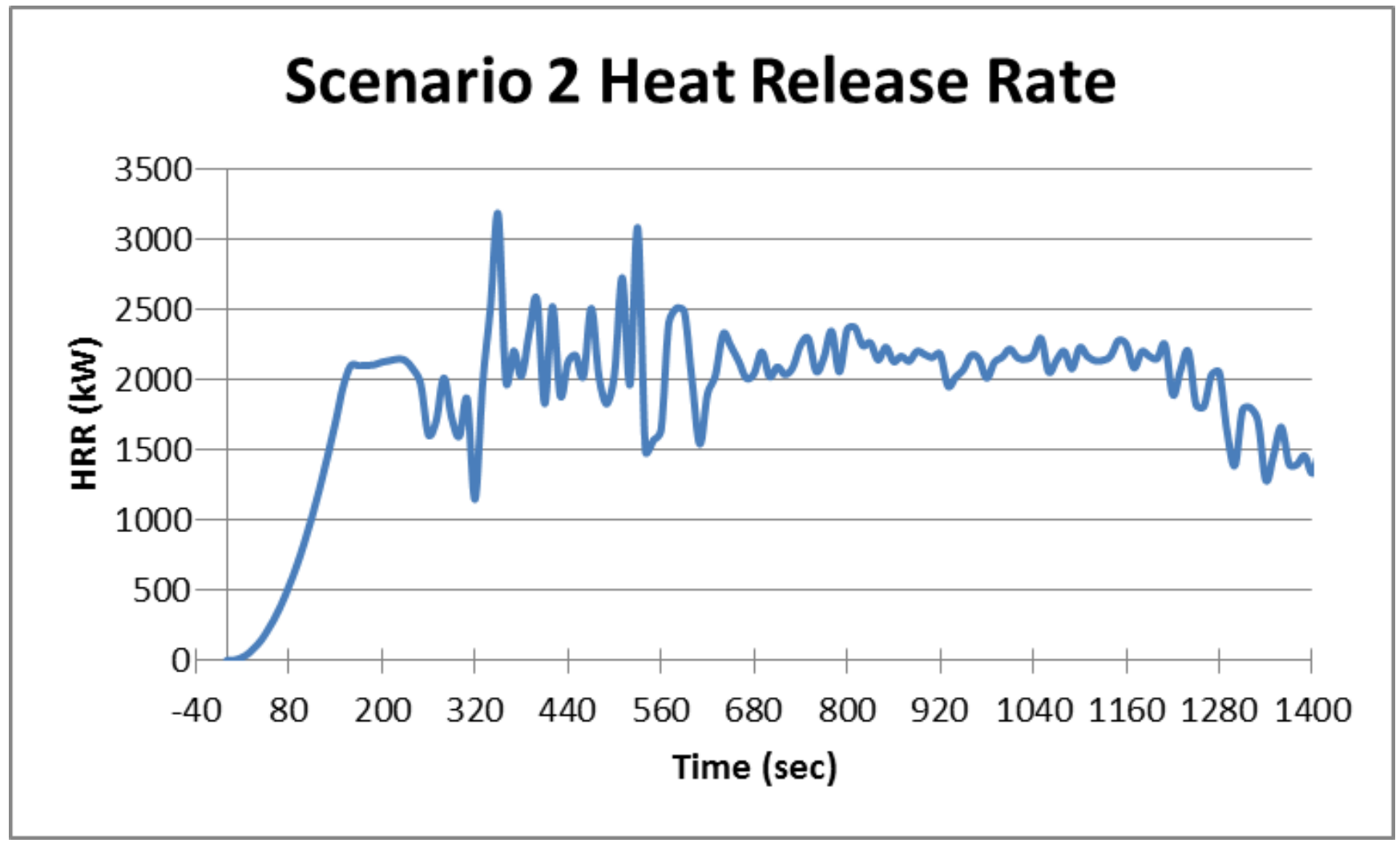

- Max Heat Release Rate: $3180 \mathrm{~kW}$ at 350 seconds 


\section{Scenario 2: Smoke Level}

Smoke Level

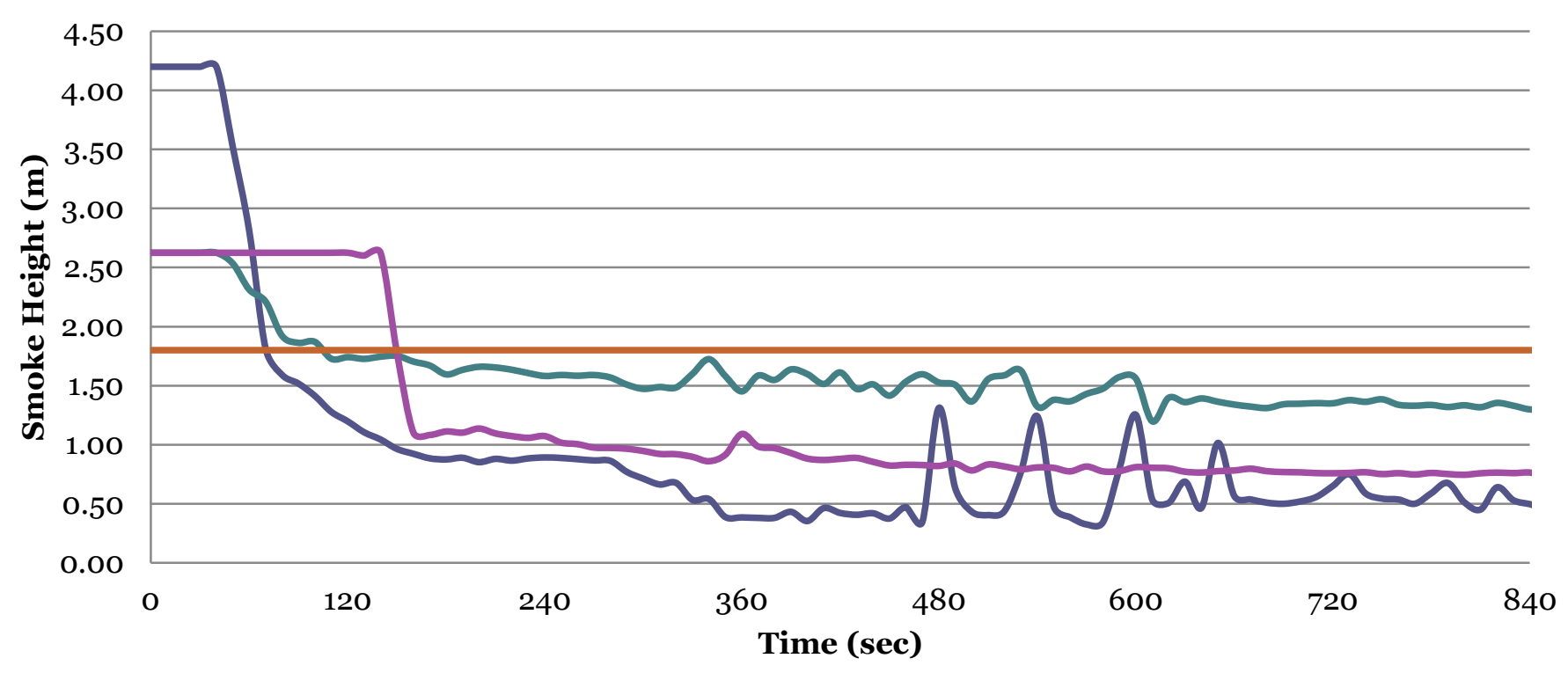

-FIRE ROOM - FIRE ROOM DOOR C CORRIDOR Criteria

- Room at 72 seconds

- Corridor at 145 seconds 


\section{Fire Scenario: Smoke Movement}

After 72 Seconds
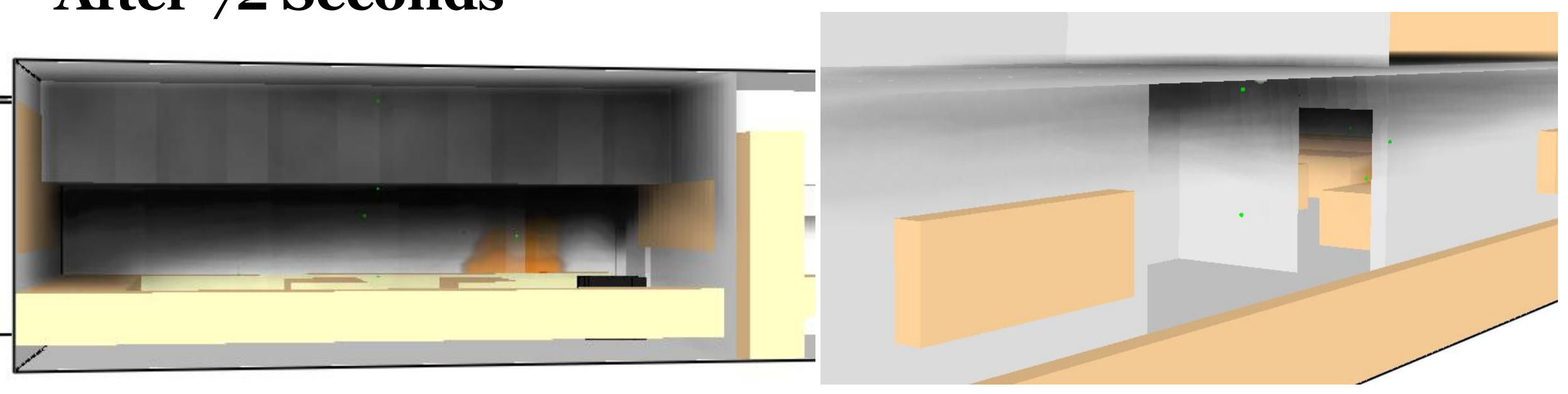

After 145 Seconds

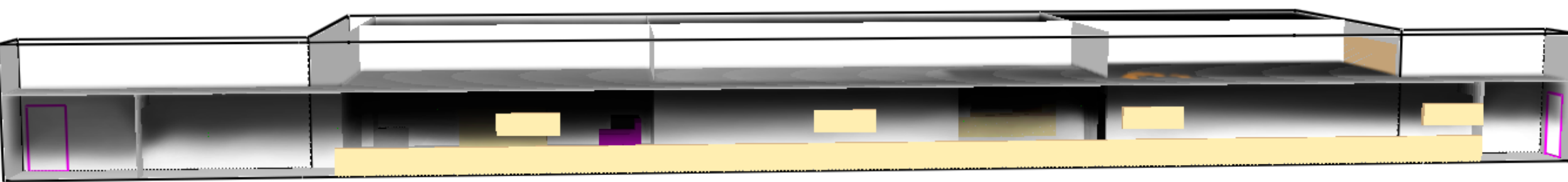




\section{Scenario 2: Visibility}

- Using Soot to approximate visibility in meters

- Optical Density

- Room at 115 seconds

- Corridor at 240 seconds

\section{After 240 seconds}

\section{After 115 seconds}
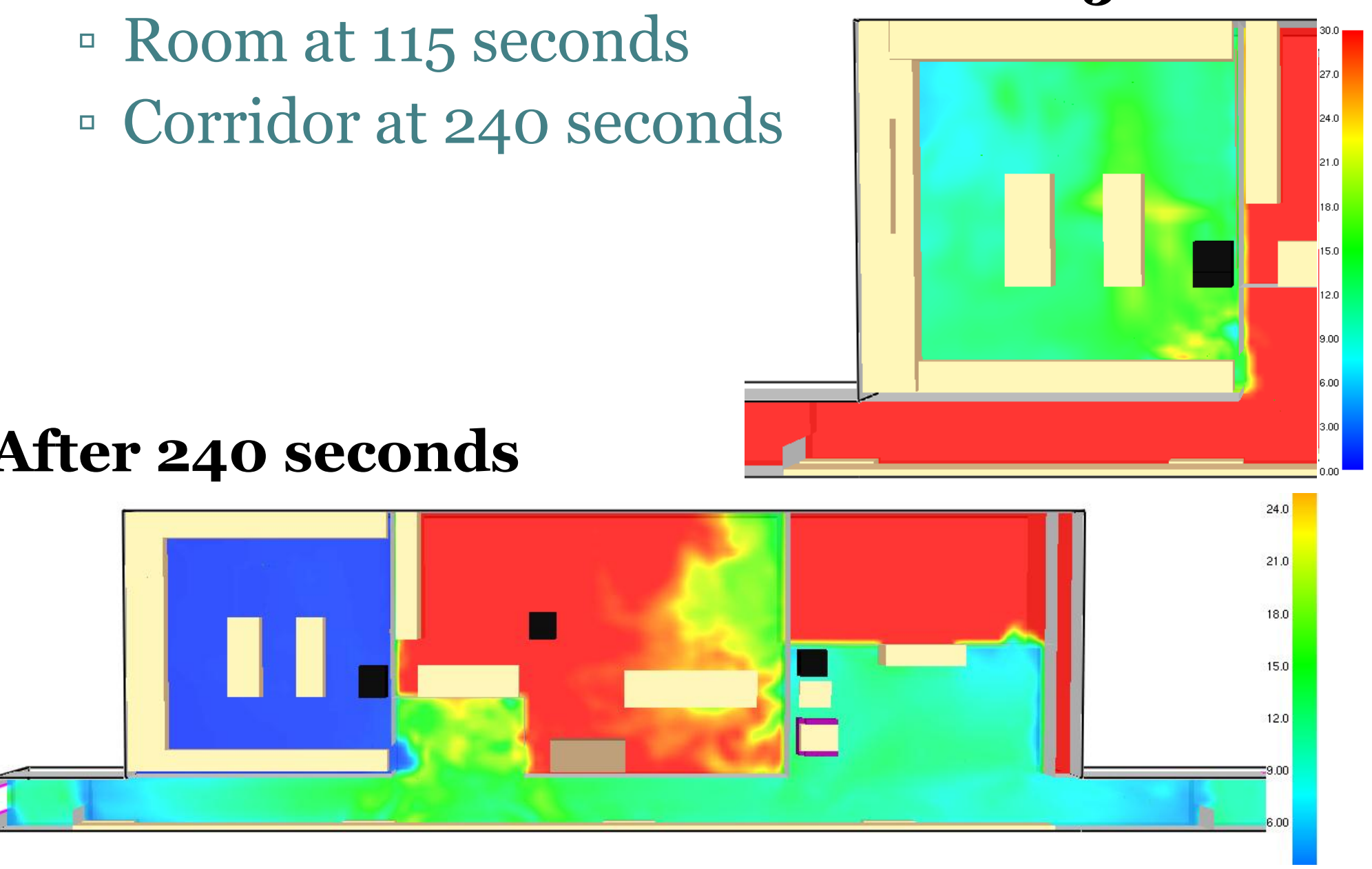


\section{Fire Scenario: 3}

- Fourth Floor

- Pinch point in the T in the floor plan

- There is a fire door propped open that leads to a dead-end

- Not analyzed based on time limitations and significance

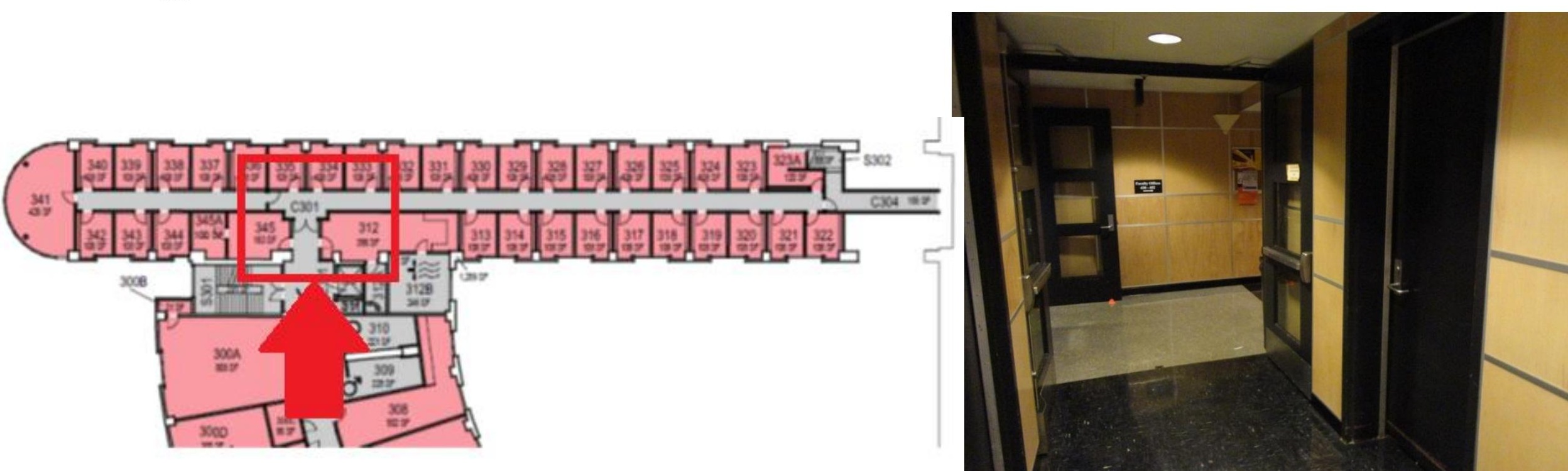




\section{Egress Analysis}

- Entire Building Evacuation

\begin{tabular}{|c|c|c|c|c|c|}
\hline CASE & $\begin{array}{c}\text { Hand } \\
\text { Calculation } \\
\text { SFPE } \\
\text { Estimate } \\
\text { (min) }\end{array}$ & $\begin{array}{c}\text { Hand } \\
\text { Calculation } \\
\text { Paul's } \\
\text { Estimate } \\
\text { (min) }\end{array}$ & $\begin{array}{c}\text { Pathfinder } \\
\text { Estimate } \\
\text { (min) steering } \\
\text { mode }\end{array}$ & $\begin{array}{c}\text { Pathfinder } \\
\text { Estimate } \\
\text { (min) SFPE } \\
\text { mode }\end{array}$ \\
\hline $\mathbf{1}$ & $\begin{array}{c}\text { All exits } \\
\text { available }\end{array}$ & 5.4 & 5.4 & 3.4 & 4.5 \\
\hline $\mathbf{2}$ & $\begin{array}{c}\text { Only Vertical } \\
\text { Exit Available }\end{array}$ & 9.0 & 7.9 & 5.0 & 6.2 \\
\hline $\mathbf{3}$ & \begin{tabular}{c} 
Silo \\
\hline
\end{tabular} & 2.5 & 4.2 & 1.1 & 1.7 \\
\hline
\end{tabular}




\section{Travel Time: Fourth Floor}

SFPE Method: 49 Seconds Steering Mode: 70 Seconds Hand Calculation SFPE: 80 Seconds

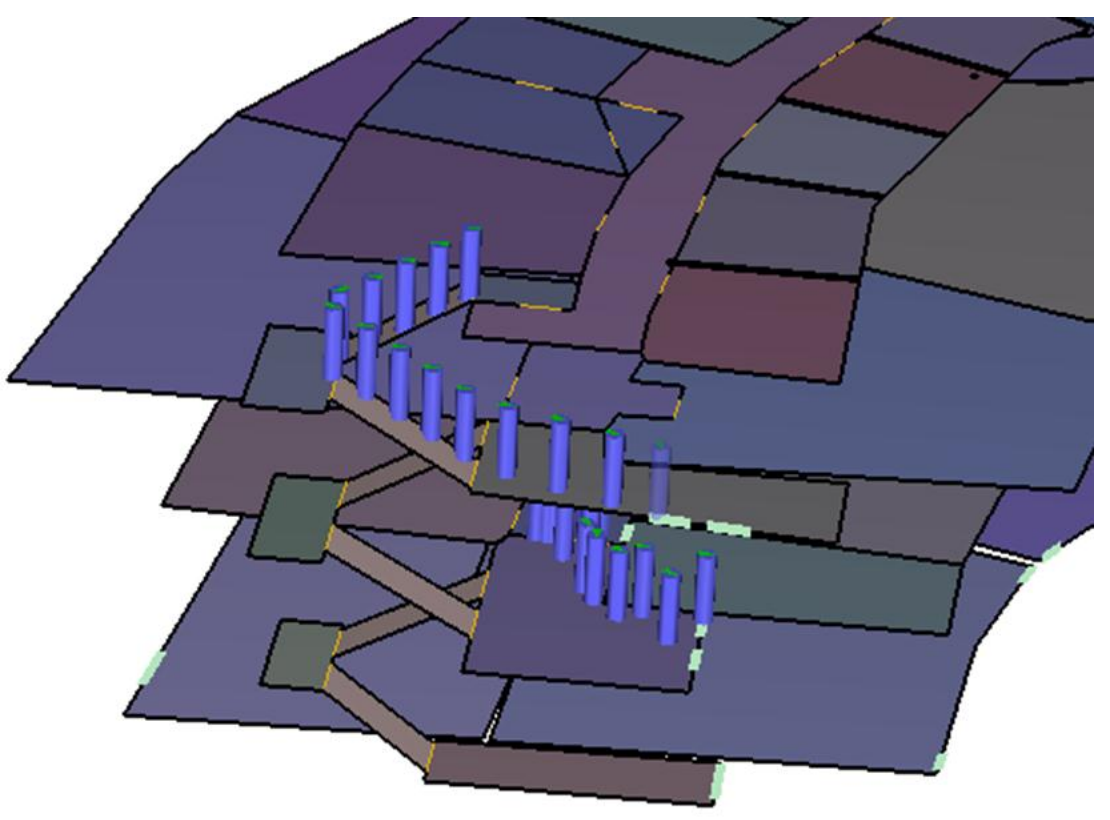

Evacuation of fourth Floor 70 seconds 


\section{RSET vs. ASET}

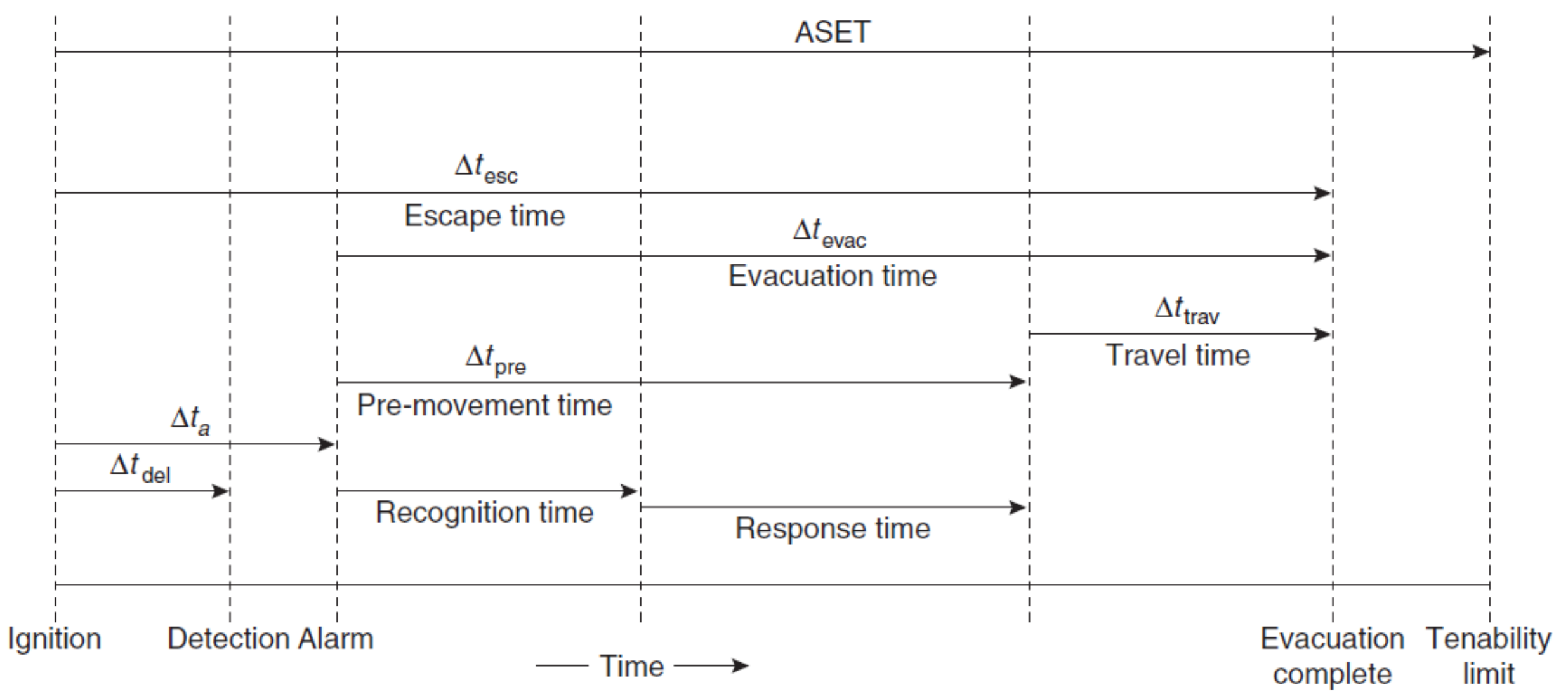

Mowrer, F. W., \& Rosenbaum, E. R. (2008). Fire Protection Handbook Information and Analysis for Fire Protection. 


\section{RSET}

- Time to Alarm:

- Occupants can detect a fire much quicker than detection system

- If detected by human or manual means: time to detection + time to activate the pull station

- Comparable to Smoke detection activation

- Scenario 1

- Smoke detection activation 30 seconds

- Scenario 2

- Smoke Detection Activation: 110 seconds 


\section{RSET Assumptions}

- Pre-movement Time

- Occupants:

- Familiar with their surroundings

- Trained staff member in every classroom

- Occupants preference to be elsewhere

- Belongings are mobile

- Mild climate

- False alarms

- NFPA HB and SFPE HB estimates

- Assume 45 seconds based on these factors 


\section{Conclusions and Recommendations}

\begin{tabular}{|c|c|c|c|c|}
\hline \multicolumn{5}{|c|}{ RSET } \\
\hline & & Limit & Scenario 1 (sec) & Scenario 2 (sec) \\
\hline \multirow{4}{*}{$\begin{array}{c}\text { Mainain tenible } \\
\text { conditions in corridor }\end{array}$} & $\mathrm{CO} \mathrm{ppm}$ & 1,400 & 850 & 1160 \\
\hline & Temperature (c) & 60 & 82 & 105 \\
\hline & Optical density $(1 / \mathrm{m})$ & 0.2 & 75 & 240 \\
\hline & Smoke level (m) & 6.1 & 90 & 145 \\
\hline $\begin{array}{l}\text { Prevent Flashover in } \\
\text { room of origin }\end{array}$ & Temperature (c ) & 600 & - & 135 \\
\hline \multicolumn{5}{|c|}{ ASET } \\
\hline & \multicolumn{2}{|c|}{ Scenario 1 (sec) } & \multicolumn{2}{|c|}{ Scenario $2(\mathrm{sec})$} \\
\hline Time to Alarm & \multicolumn{2}{|c|}{30} & \multicolumn{2}{|r|}{110} \\
\hline Pre-Movement Time & \multicolumn{2}{|c|}{45} & \multicolumn{2}{|r|}{45} \\
\hline Travel Time & \multirow{2}{*}{\multicolumn{2}{|c|}{70}} & \multicolumn{2}{|r|}{70} \\
\hline Total & & 145 & \multicolumn{2}{|c|}{225} \\
\hline
\end{tabular}




\section{Scenario 1: Performance Criteria Bar Graph}

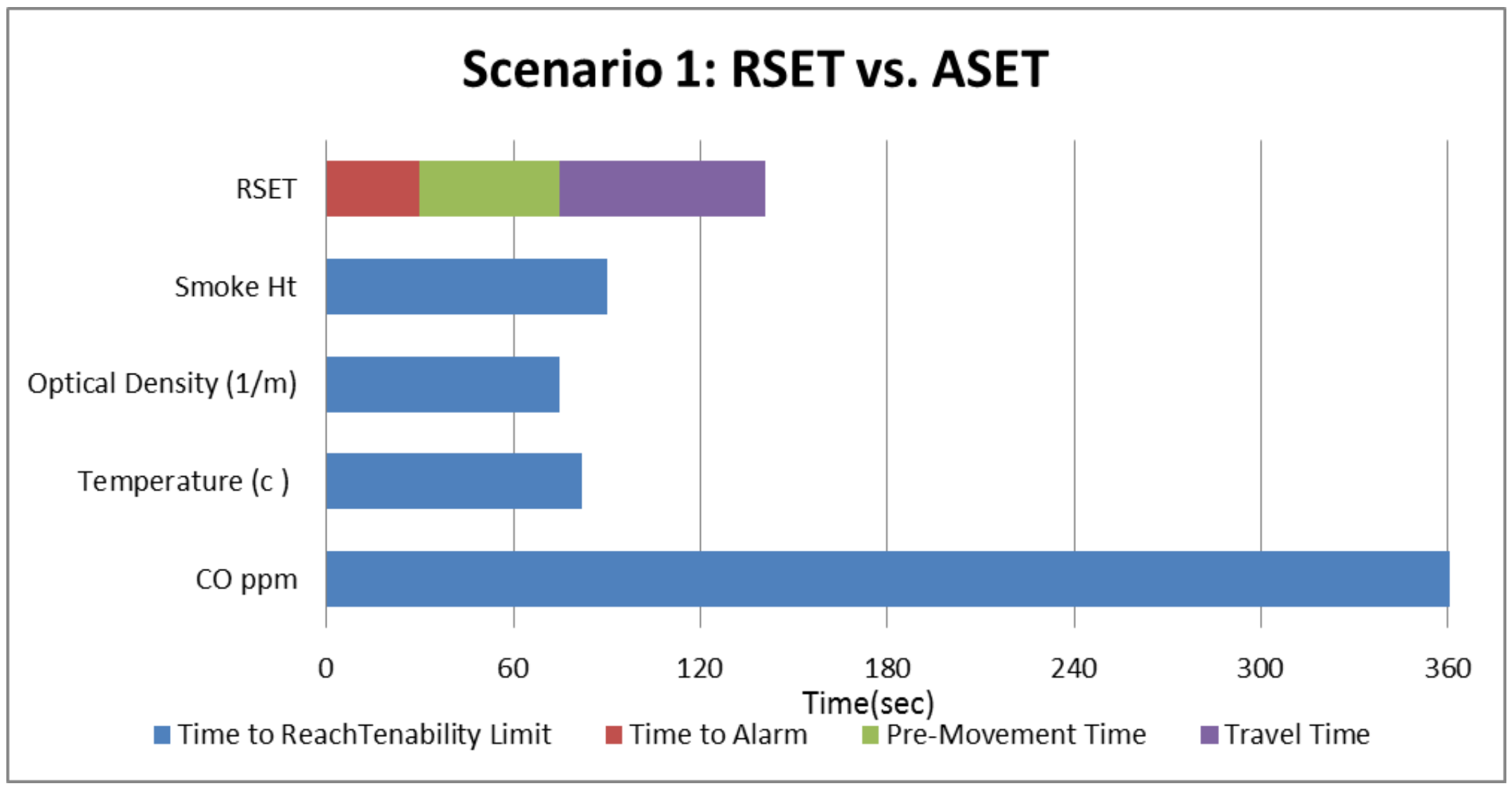




\section{Scenario 2: Performance Criteria Bar Graph}

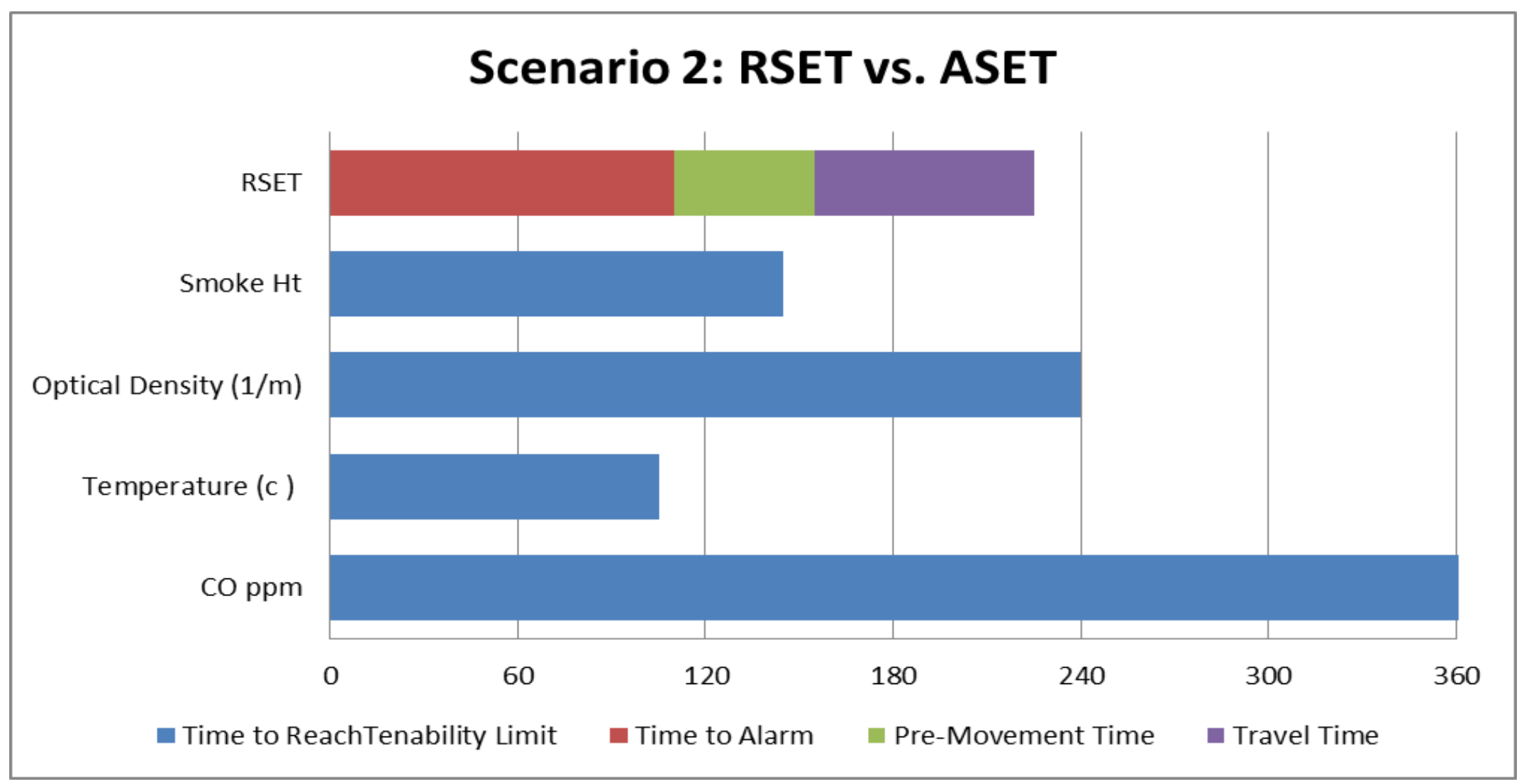




\section{Recommendations}

- Automatic Fire Sprinklers

- Containment/Suppression of Fire

- Damage to Property

- Cost Benefit Analysis

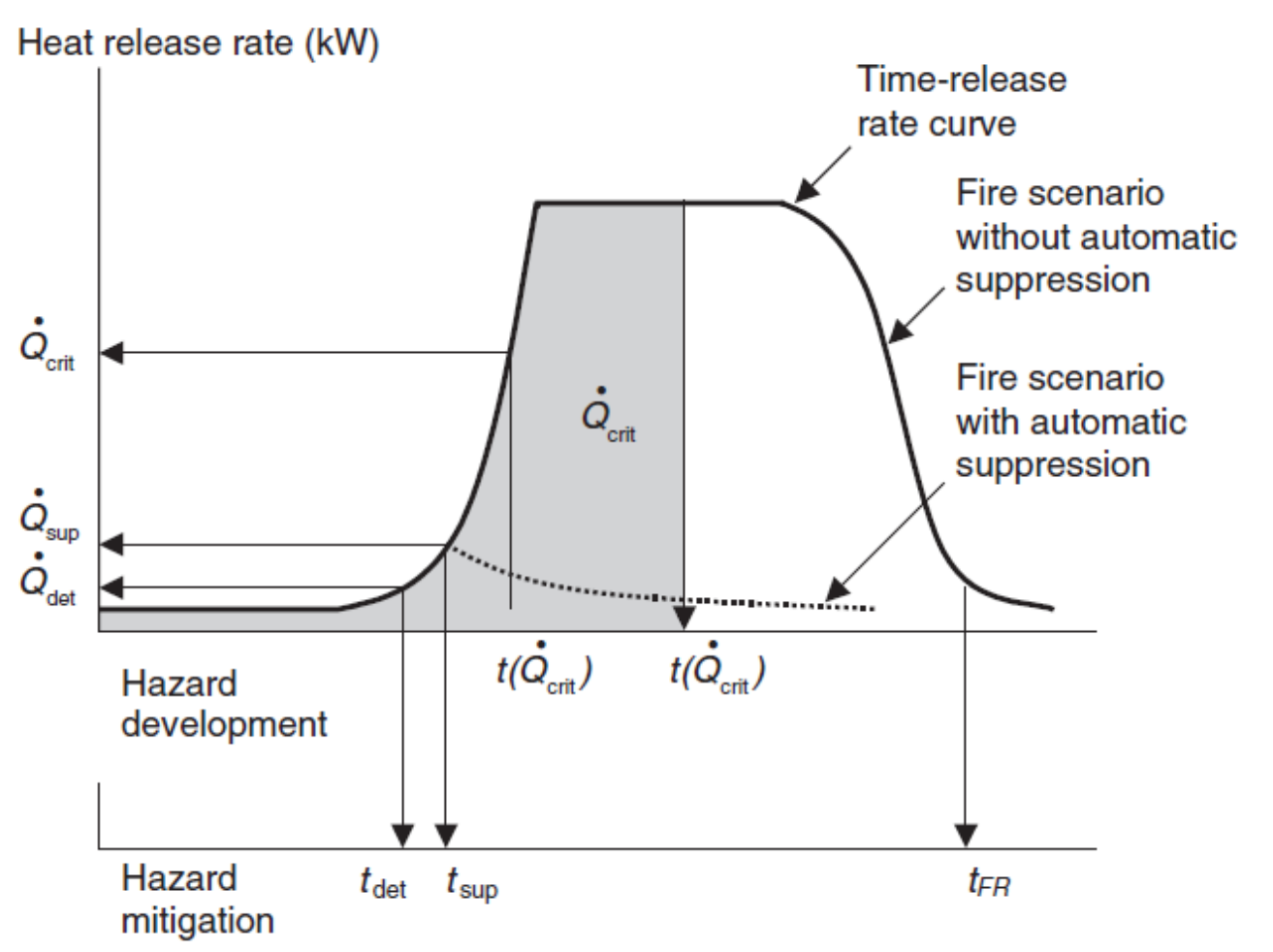

Time scales $\longrightarrow$

Mowrer, F. W., \& Rosenbaum, E. R. (2008). Fire Protection Handbook, 3-11 Overview of Performance-Based Fire Protection Design 


\section{Recommendations}

- Upgrade Fire Alarm and Detection System

- Reduce Detection Time

- Improve Notification Appliances

- Fire Management Plan

- Regulate Fire Hazards

- Eliminate Hazardous Conditions

- Inspection, Testing and Maintenance 


\section{Q\&A}

Special Thank You To:

- Professor Christopher Pascual

- Professor Frederick Mowrer

- Professor Thomas Korman

- Professor Lonny Simonian

- Professor David Rich

- Professor Lautenberger

- Professor Daniel Gemeny

- Professor John Chen 